OPEN ACCESS

Edited by:

Simone Brogi,

University of Pisa, Italy

Reviewed by:

Xuewei Zhu,

Wake Forest School of Medicine,

United States

Charles E. McCall,

Wake Forest Baptist Medical Center,

United States

${ }^{*}$ Correspondence:

Lina Yu

zryulina@zju.edu.cn

Specialty section:

This article was submitted to

Medicinal and Pharmaceutical

Chemistry

a section of the journal

Frontiers in Chemistry

Received: 18 February 2021

Accepted: 01 April 2021

Published: 12 May 2021

Citation:

Lin J, Ren J, Gao DS, Dai Y and Yu L (2021) The Emerging Application of

Itaconate: Promising Molecular

Targets and Therapeutic

Opportunities.

Front. Chem. 9:669308

doi: 10.3389/fchem.2021.669308

\section{The Emerging Application of Itaconate: Promising Molecular Targets and Therapeutic Opportunities}

\author{
Jiaqi Lin, Jinxuan Ren, Dave Schwinn Gao, Yi Dai and Lina Yu*
}

Department of Anesthesiology, The Second Affiliated Hospital, Zhejiang University School of Medicine, Zhejiang University, Hangzhou, China

Metabolites have recently been found to be involved in significant biological regulation and changes. Itaconate, an important intermediate metabolite isolated from the tricarboxylic acid cycle, is derived from cis-aconitate decarboxylation mediated by immune response gene 1 in mitochondrial matrix. Itaconate has emerged as a key autocrine regulatory component involved in the development and progression of inflammation and immunity. It could directly modify cysteine sites on functional substrate proteins which related to inflammasome, signal transduction, transcription, and cell death. Itaconate can be a connector among immunity, metabolism, and inflammation, which is of great significance for further understanding the mechanism of cellular immune metabolism. And it could be the potential choice for the treatment of inflammation and immune-related diseases. This study is a systematic review of the potential mechanisms of metabolite associated with different pathology conditions. We briefly summarize the structural characteristics and classical pathways of itaconate and its derivatives, with special emphasis on its promising role in future clinical application, in order to provide theoretical basis for future research and treatment intervention.

Keywords: itaconate, itaconate derivative, inflammation, immunometabolism, therapy

\section{INTRODUCTION}

In the past decade, how intracellular metabolic changes control immunity and inflammation rose a resurgence of interest in immune metabolism (Kabat and Pearce, 2017). Tricarboxylic acid (TCA) cycle situates at the core of cellular metabolism and is the most common metabolic pathway in aerobic organisms (Martínez-Reyes and Chandel, 2020). TCA cycle is indispensable among many metabolic processes in activated macrophages. It can regulate the metabolic adaptability of macrophages and affect their effect. Itaconate is an important intermediate metabolite isolated from the TCA cycle, which has recently been found to participate in significant biological regulation and changes (Murphy and O'Neill, 2018).

Itaconate was first synthesized by chemical method in 1836 (Baup, 1836), and then it was confirmed that itaconate was synthesized by decarboxylation of cis-aconitate (Turner et al., 1840) and in 1955 immune response gene 1(Irg 1) was found to play an important role in mediating itaconate production in mitochondrial matrix (Lee et al., 1995). 
In fact, less was known about the molecular regulatory mechanisms behind the biosynthesis of itaconate led to its mainly application in industrial polymer synthesis since the 1950s (Rao et al., 2006). However, with the rapid development of metabolomics, an increasing number of potential mechanisms of itaconate has attracted more and more attention. The antiinflammatory and anti-immune mechanisms of itaconate were described in two 2016 Nature articles (Bambouskova et al., 2018; Mills et al., 2018). In the process of inflammatory activation of macrophages infected by pathogens or stimulated by lipopolysaccharide(LPS), the intermediate products of TCA, such as succinate, citrate, and itaconate continuously accumulated to affect the expression of inflammatory factors and the concentration could reach the millimole level (Murphy and O'Neill, 2018). Itaconate may be the best example role as a dual viability of immunomodulators on both the transcriptional and metabolic levels and has been shown to participate in variety of the initiation and maintenance of the anti-inflammatory response in macrophages (Yu et al., 2019).

Since itaconate was pushed to the limelight as a key determinant and participated in macrophage stimulation as an important regulatory metabolite. Subsequently, a large amount of researches report that itaconate is a central and determinant component links three fields of immune, metabolism and inflammation together which is of great significance for further understanding mechanism of cellular immune metabolism and drugs development for the treatment of inflammatory and immune-related diseases in the future (Hooftman and O'Neill, 2019; O’Neill and Artyomov, 2019). This article reviews the biosyhthesis, structure, metabolic characteristics, classical pathway of itaconate, and summarizes its potential role on current clinical application in order to provide a theoretical basis for future research and treatment intervention.

\section{THE BIOSYNTHESIS AND METABOLISM OF ITACONATE}

TCA cycle is a complex biological process involving a series of enzyme-catalyzed reactions for Adenosine triphosphate (ATP) production in the mitochondrial matrix (Martínez-Reyes and Chandel, 2020). The unique and crucial step in the biosynthesis pathway of itaconate is the decarboxylation of cis-aconitate produced by the dehydration of citrate. The decarboxylation is encoded by aconitate decarboxylase 1 (ACOD1), also named Irg1 (Michelucci et al., 2013). Irg1 overexpression leads to increased synthesis of endogenous itaconate (Wu et al., 2020). Catalytic conversion of isocitrate to $\alpha$-ketoglutarate dehydrogenase further causes accumulation of citrate (itaconate precursor) (Nonnenmacher and Hiller, 2018). In addition, pyruvate dehydrogenase complex (PDC) catalyzes and controls the irreversible conversion of pyruvate into citrate precursor acetyl-CoA (Michelucci et al., 2013). Phosphorylation of PDC by pyruvate dehydrogenase kinase 1 (PDK1)inactivates the enzyme and reverses this conversion (Denko, 2008; Meiser et al., 2016). Itaconate can be metabolized into itaconyl-coenzyme A (CoA), which inactivates mitochondrial CoA B12, resulting in a decrease in the activity of methylmalonyl-CoA mutase (MUT) and production of MUT-dependent branched-chain amino acids(BCAAs) (Zhu et al., 2020; Cordes and Metallo, 2021). Itaconate and its metabolite can affect other energy metabolism pathways besides the TCA cycle. The biosynthesis and metabolism of itaconate were shown as Figure 1.

\section{GENERAL FEATURES OF ITACONATE}

The lack mechanistic details of itaconate biology and regulatory function has led to the synthesis of several cell-permeable derivatives of itaconate, such as dimethyl itaconate (DI),4-octyl itaconate (4-OI) and ethyl itaconate (4-EI) to imitate the action characteristics of endogenous Itaconate. Thus, this section sets out to perform an introduction of the metabolic, electrophilic, and immunological properties of the itaconate and its three major derivatives reported in the literature to date: Itaconate, DI, 4OI, and 4-EI (Table 1).

\section{Itaconate}

Itaconate is an $\alpha, \beta$-unsaturated dicarboxylic acid (C5H6O4) containing a double bond and two carboxyl groups (Robert and Friebel, 2016). It is an electrophile which has active chemical properties through forming a bond with a nucleophile by accepting an electron pair and to some extent showed similar characteristics to underlying enzymatic mechanisms (Robert and Friebel, 2016). Because of the conjugated unsaturated double bond structure of itaconate, it can affect the activity and function of substrate proteins by covalently modification of the cysteine residues in proteins in the way of Michael addition reaction, thereby exerting a strong potential in inhibitory effects on the inflammatory signaling pathway (Bambouskova et al., 2018; Swain et al., 2020). However, the high polarity and low electrophilicity of unmodified itaconate lead to its weak cell-permeability to satisfy further research. Previous work on itaconate are based on its derivatives. To better understand the real characterize of itaconate, Wang et al. designed a biorthogonal probe -Itaconate-alkyne (ITalk) in 2020 which retain the $\alpha, \beta$-unsaturated carboxylic acid group and the long carbon chain (Qin et al., 2020). It was designed to directly capture modified proteins in living cells to identify bona fide targets of itaconate on a large scale. One thousand one hundred thirty-one itaconate-modified cysteine sites belonging to 1,926 itaconate-modified proteins including 199 hypersensitive targets were identified, including the previously known functional substrate proteins Aldolase A (ALDOA) and Kelch like ECHassociated protein 1 (Keap1) (Qin et al., 2019, 2020). Many key proteins heavily modified such as inflammasome, signal transduction, transcription, and cell death- related proteins involved in inflammatory immune response and host defenserelated regulatory pathways suggesting that itaconate could affect macrophage function by regulating multiple pathways. This work greatly expanded a valuable resource database of biological roles of itaconate for further investigation. 


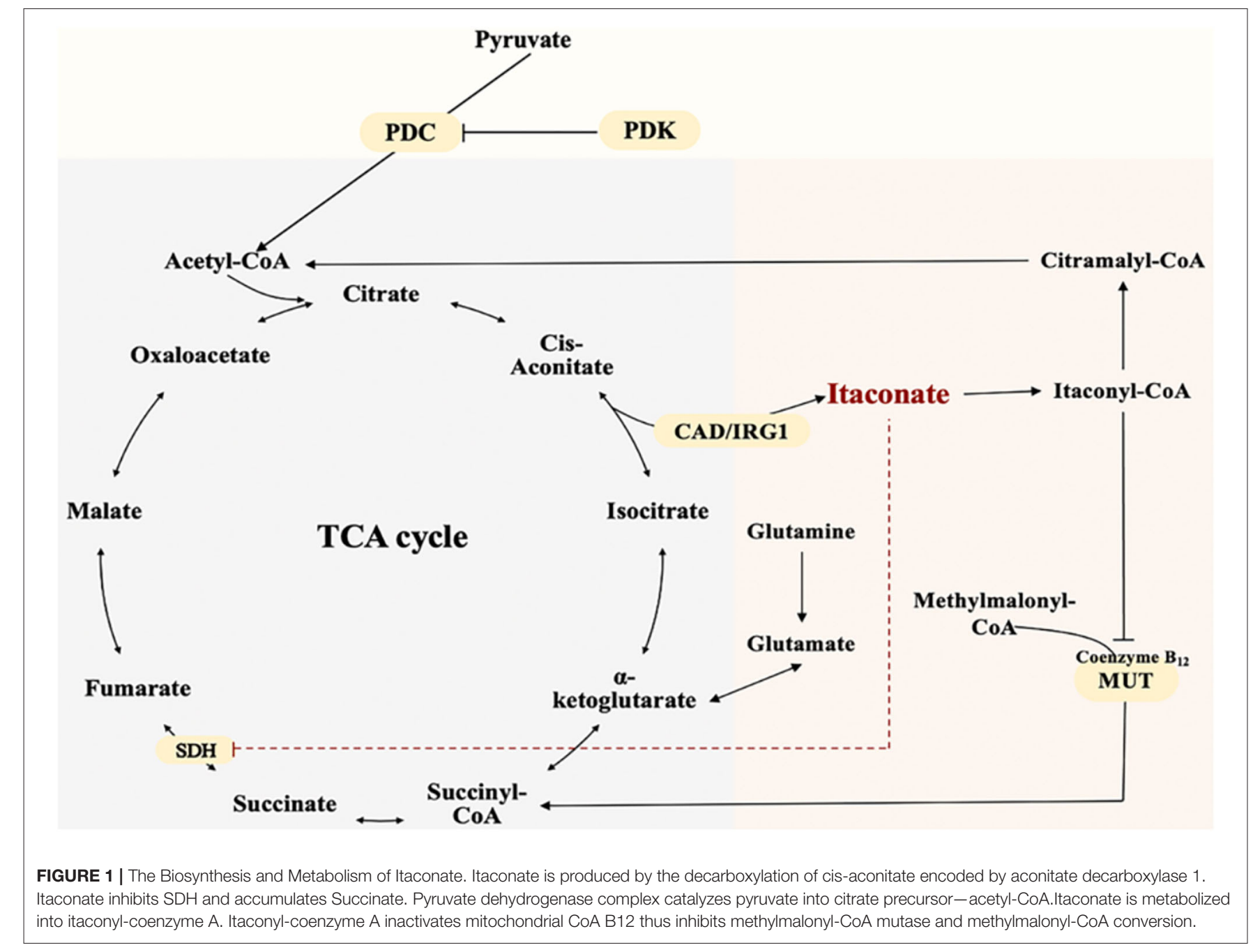

TABLE 1 | The chemical structures of itaconate and its derivatives.

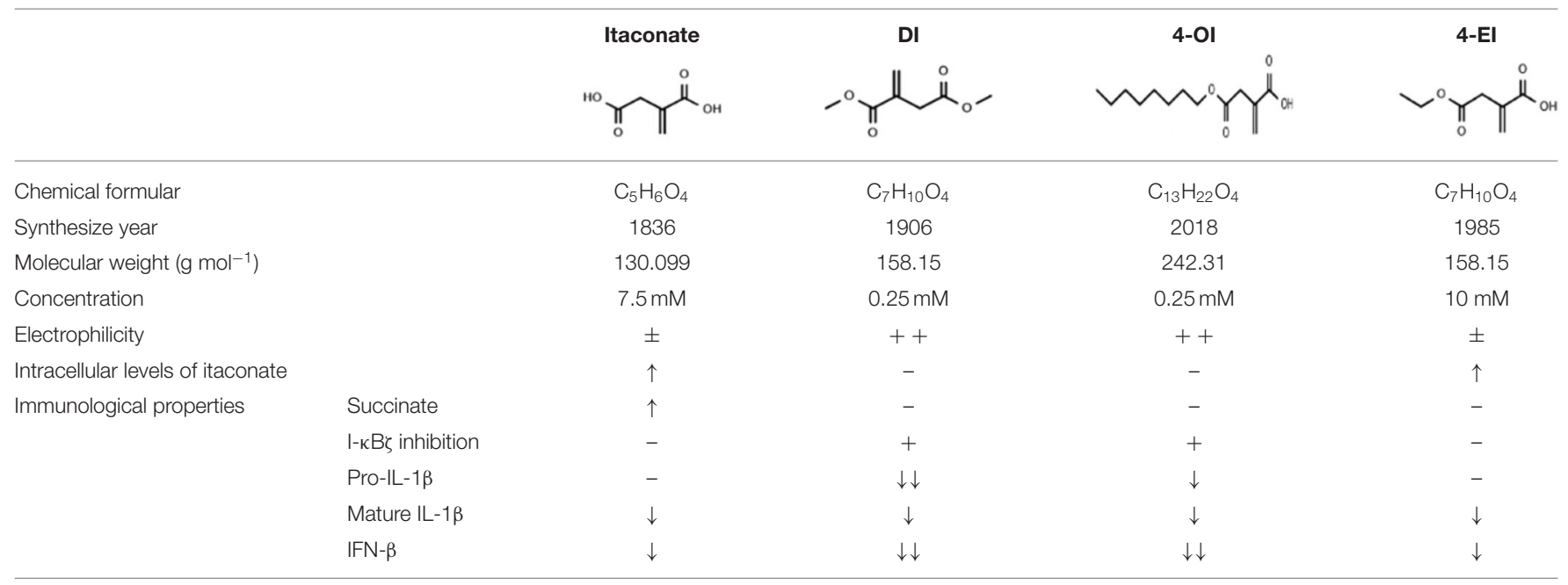

The chemical structures, molecular weight, electrophilicity and properties for itaconate and its derivatives a are summarized in this table. 


\section{Dimethyl Itaconate}

Itaconate derivative dimethyl itaconate (DI) first employed as a chemical experimental material is considered as "poweredup" version of itaconate (RajanBabu et al., 1999; Schmidt et al., 2008). The membrane-permeable ability of DI is attributed to a carboxyl group esterification on the 1-position (Lampropoulou et al., 2016). The conjugation effect of ester group prevents electron transfer and reduces the negative charge of itaconate and render its high acceptor reactivity in Michael addition. This is also the reflection DI significantly and acutely deplete glutathione (GSH) levels. The structural characteristics of DI makes it as a thiol-reactive metabolite to induce nuclear transcription factor erythroid-2-related factor 2 (Nrf2) and several Nrf2 target genes in a similar manner to Dimethyl fumarate (DMF), another potent Nrf2 activator (Carlstrom et al., 2019). The ability of DI to block LPS-induced IKBל expression is the convincing proof of its strong electrophilicity (Bambouskova et al., 2018). However, in recent studies showed that short-term effect because of rapidly degradation or further metabolization of DI was not enough to release endogenous itaconate, hence is unlikely to be converted to intracellular itaconate (ElAzzouny et al., 2017). It could be inferred DI could not mimic itaconate and the broad metabolic effects by DI was not caused by intracellular itaconate accumulation but electrophilicity and covalent modification of metabolic enzymes (Swain et al., 2020). However, under the stimulation of LPS, treated with increasing concentrations of exogenous DI can increase the biosynthesis of itaconate (ElAzzouny et al., 2017).

\section{4-Octyl Itaconate}

Esterification on the 1-position of DI is a direct effect on the rapidly intracellular thiols reaction of $\mathrm{Nrf} 2$. And to overcome the limitations of DI, Mills designed a new itaconate surrogate, 4octyl itaconate (4-OI) (Mills et al., 2018). The difference between 4 -OI and DI is the location of octyl ester. Octyl ester group of 4-OI is located distal to the alkene than DI which makes 4OI lower thiol reactivity similar to itaconate and more suitable to probe the physiological function of itaconate (Mills et al., 2018; Sano et al., 2020). The structural differences also made 4-OI lacks the electrophilic property which makes it a better candidate of Itaconate (Ryan et al., 2019). 4-OI can increase the level of itaconate by the way of hydrolyzation with or without the stimulation of LPS (Swain et al., 2020). This is the significant difference between DI and 4-OI, as previous discussion, DI only increase the biosynthesis of itaconate but cannot convert into intracellular itaconate (ElAzzouny et al., 2017). The main reason is the long carbon chain of 4-OI compared to DI to resistant to esterase hydrolysis. However,4-OI is better to mimic endogenous itaconate but not perfect enough. There are still difference such as hydrophobicity between itaconate and 4-OI on spectrum of proteins modify (Mills et al., 2018). All of these data suggest that the derivatives of itaconate mediate diverse pathways to protect against macrophage inflammation. Further studies should be explored for the extra side functions of DI and 4-OI.

\section{4-Ethyl Itaconate}

At present, there are few researches on 4-ethyl itaconate (4-EI), in an article comparing the metabolic, electrophilic, and immunologic profiles of itaconate and its derivatives has mentioned 4-ethyl itaconate (4-EI) (Swain et al., 2020). Esterified carbonyl group enhances the negatively charged, accordingly, this is why DI could induce a strong electrophilic stress response than that of 4-OI and endogenous itaconate, and makes DI play a better role of cell permeability (Mills et al., 2018; Swain et al., 2020). 4-EI has similar structure to DI, the electrophilicity of 4EI is lower but polarity is higher than that of DI because only 4-carboxyl of 4-EI is esterified. It is also reflected in that 4-EI has no inhibitory effect on IкBל. However, when given butylthionine sulfoxide (BSO), a non-electrophilic inhibitor of GSH synthesis, 4-EI has a consistent effect with DI on transcription factor

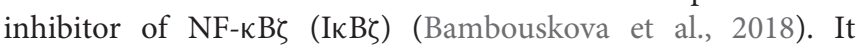
probably because the existence of GSH lead to a substantial decrease in 4-EI or DI reactivity. Another ethyl itaconate -1ethyl itaconate(1EI) which the first position carboxyl group is esterified also make the inhibitory effects on ІкBל. Structural differences of different ester groups at different positions also correlate with functional differences (Bambouskova et al., 2018).

The study of itaconate as therapeutic molecules has generated excellent prospects in the pharmaceutical industry due to its low toxicity and high biological activity. None of the above three derivatives can well-simulate the ibona fide targets of itaconate, so there is an urgent need for a more perfect derivative to study the mechanism of itaconate more comprehensively.

\section{THE CLASSICAL MECHANISMS OF ITACONATE}

At present, there are two kinds of studies on itaconate: $\operatorname{Irg} 1^{-/-}$ macrophages and the regulatory effect of itaconate derivatives. These two results were complementary and revealed that the regulatory mechanisms of itaconate involved alkylation on Keap1 to activate Nrf2, succinate dehydrogenase inhibition, activating transcription factor 3 (ATF3) induction to inhibit IкB $\zeta$ activation, down-regulating glycolysis by GAPDH and ALDOA alkylation. The electrophilicity of itaconate and its derivatives are also indispensable in the process of metabolic regulation. Here, we will conclude the classical mechanism of itaconate to clarify its potential targets (Figure 2).

\section{Transcriptional Regulation Irg1-Nrf2-Keap1}

Nrf2 act as a multifunctional and indispensable player in modulating the inflammatory response and oxidative stress (Yang et al., 2014; Yamamoto et al., 2018). In the physiological homeostatic condition, unstimulated Kelch like ECH-associated protein 1 (Keap1) as a repressor combined with Nrf2 and sequestrated Nrf2 in the cytoplasmic (Cuadrado et al., 2019). Keap1 also facilitates Nrf2 degradation by ubiquitin-mediated proteasomal degradation pathway (Villeneuve et al., 2010). Mechanistically, Itaconate due to its electrophilicity structure 


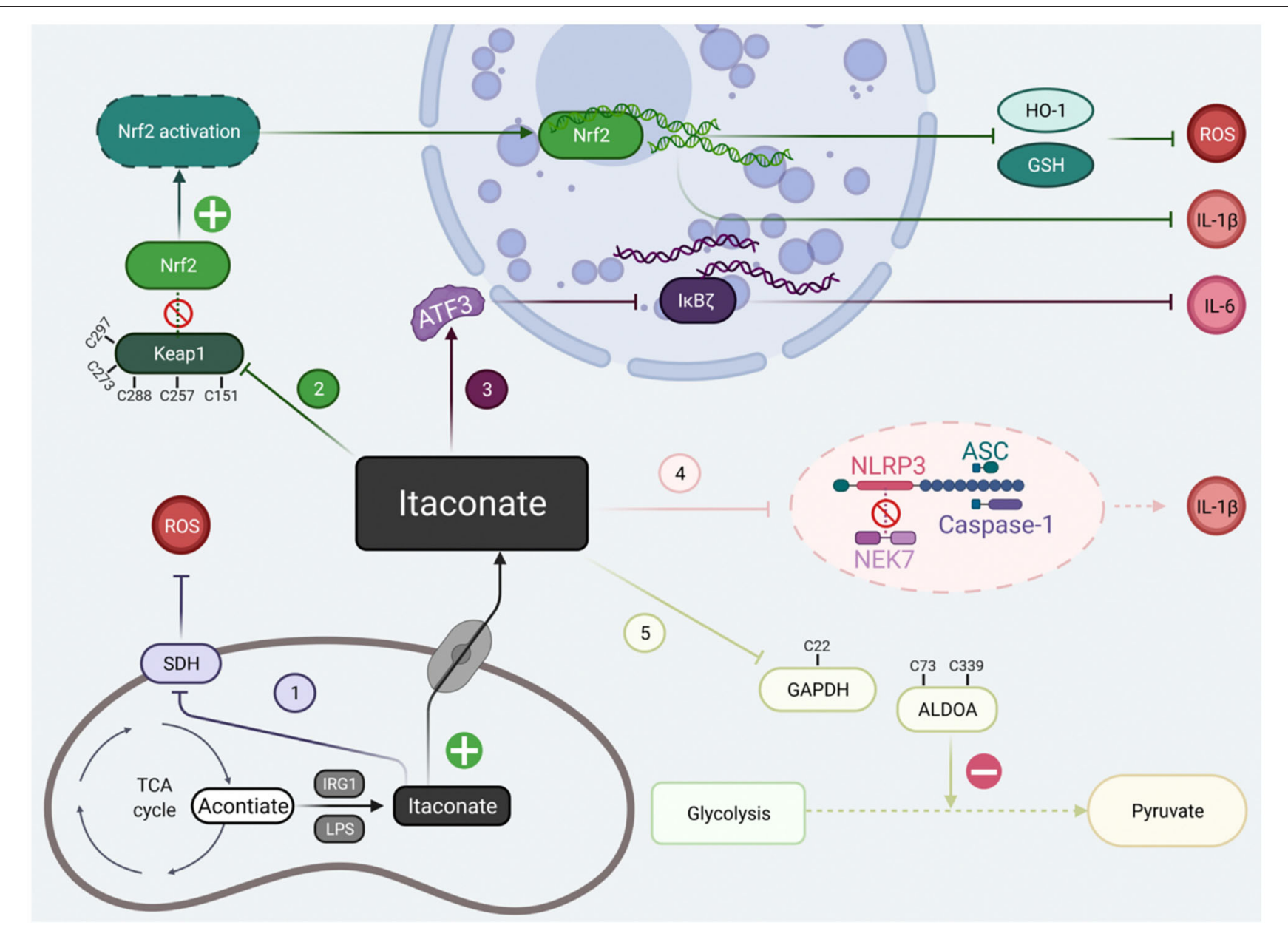

FIGURE 2 | The classical signal pathways of itaconate that have been studied at present. The classical signal pathways of itaconate can be divided into five main types. (1) Itaconate mediated by IRG1 could inhibit due to structural similarity with succinate. (2) Itaconate covalently modify Keap1 cysteine 151 etc.to dissociate the combination of the Keap1-Nrf2, thus promote migration of Nrf2 to cell nuclei. (3) Itaconate increases the levels of ATF3 protein which translocated to the cell nuclei to inhibit $I_{\kappa} B \zeta$ at the translational level. (4) Itaconate abolish NLRP3-NEK7 connection in a modification termed dicarboxypropylation on C548 of NLRP3 thus block NLRP3-dependent IL-1 $\beta$ release. (5) Itaconate inhibit glycolysis by alkylating cysteine 22 residues on GAPDH, cysteine 73, and 339 on ALDOA. Created with Biorender.

of containing $\alpha, \beta$-unsaturated carboxylic acid could make it form a modification of 2,3-dicarboxypropyl adduct to covalently modify cysteine 151 (C151) residues on KEAP1 by a Michael addition (Mills et al., 2018; Ryan et al., 2019). Alkylation of Keap1 cysteine residues disassociates the combination of Keap1-Nrf2 and liberates Nrf2 accumulated and translocated to the cell nuclei to initiate transcription of anti-inflammatory and anti-oxidant program. This opens the possibility that itaconate might extend the scope of the biological therapies by cysteine modification of numerous target proteins. Moreover, the effectiveness of Nrf2 regulator in response to LPS was chopped in Irg1- deficient macrophages (Mills et al., 2018).

\section{ATF3- IкB $\zeta$}

The mechanism of inflammation activation is a complex and continuous multi-step process. Except for Nrf2-dependent transcriptional regulation, a unique anti-inflammatory action of itaconate targets on $\mathrm{ATF} 3-\mathrm{I} \kappa \mathrm{B} \zeta$ pathway in a Nrf2-independent manner to mediate the inflammatory response (Bambouskova et al., 2018). ІкB $\zeta$ is a nuclear protein encoded by Nfkbiz gene that could control the release of proinflammatory cytokines interleukin-6 (IL-6), a product of the secondary but not primary transcriptional program responses to LPS on macrophages (Ghosh and Hayden, 2008). ATF3 is negative regulator of I $\mathrm{B} \zeta$, briefly, itaconate was posited to increase the levels of ATF3 protein which subsequent inhibit the expression of

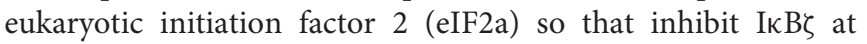
the translational level (Bambouskova et al., 2018). Itaconate provides an additional link between metabolic perturbations and inflammatory signaling by targeting ATF3- I $\mathrm{B} \zeta$ pathway.

\section{Metabolic Regulation TCA Cycle Inhibition}

Succinate dehydrogenase(SDH) also called mitochondrial complex II (CII) is an essential component for TCA cycle and cellular respiration via the electron transport chain (Mills 
et al., 2016; Bezawork-Geleta et al., 2017). SDH can oxidize and converts succinate to fumarate and eventually malate. The oxidation of succinate ultimately generates superoxide anion-reactive oxygen species (ROS), one of the most important targets of inflammation and oxidative stress (Tannahill et al., 2013; Huang et al., 2019). Itaconate was first demonstrated to inhibit SDH in 1949 (Ackermann and Potter, 1949) and further study showed succinate accumulation caused by itaconate put pro-inflammatory activated macrophages into a holding pattern (Cordes et al., 2016). Work by Lampropoulou et al. revealed that itaconate could directly block the enzyme activity of SDH using the biochemical assays (Lampropoulou et al., 2016). The main reason of SDH active site blockage arised from itaconate structural similarity with succinate and classical antagonist of SDH -malonate. Cordes et al. found that SDH inhibition by itaconate was reversible and occurred within seconds. SDH might be an early target of itaconate to affect metabolism and cell function rapidly (Cordes and Metallo, 2021). Overall, the anti-inflammation effect of itaconate by targeting on SDH strongly supports a prominent regulatory link between metabolism regulation and inflammation.

\section{Glycolysis Inhibition}

LPS stimulation changed the immunophenotype of macrophages to pro-inflammatory $\mathrm{M} 1$ and up-regulated glycolysis. Macrophages in the state of inflammation activation need to respond rapidly to stimulation by absorbing large amounts of glucose to produce abnormal bioenergy activity through glycolysis (Russell et al., 2019). Glyceraldehyde-3-phosphate dehydrogenase $(\mathrm{GAPDH})$ as a rate-limiting enzyme in the sixth step of aerobic glycolysis regulates the total rate of the whole metabolic pathway. According to previous research, Endogenous fumarate with the similarity electrophilic $\alpha, \beta$ unsaturated structural as itaconate could succinate the GAPDH and inhibit the enzymatic activity of GAPDH and thus inhibit inflammation progression (Blatnik et al., 2008). On the base of this similarity, further work by Liao et al. (2019) found the direct evidence that 4-OI directly alkylate cysteine 22 (C22) residues on GAPDH by similar dicarboxypropylation like Keap1 alkylation using the detection of U13C glucose tracing. The glycolytic blockage of 4-OI on GAPDH reduced the extracellular acidification rate and increased the intracellular oxygen consumption thereby activated the anti-inflammatory program and alleviated inflammation. Another research with the application of specific cysteine labeled probe have identified 260 itaconate-modified cysteines and found that itaconate could inhibit glycolysis by a negative feedback regulation on another two key enzymes to resist inflammation (Qin et al., 2019). Alkylation of two cysteines (Cys73 and Cys339) of ALDOA by itaconate have the same effect on the inhibition to impair glucose catabolism. However, alkylation of Cys84 of lactate dehydrogenase (LDHA) have more effect on lactate production than glucose accumulation. It probably because ALDOA controls the first step of glycolysis catalyzed reaction whereas LDHA convers pyruvate to lactate at the last step (Qin et al., 2019, 2020). This mechanism offers a novel insight of intracellular metabolites in the complex regulation function of inflammation progression.

\section{THE CANDIDATE MECHANISMS OF ITACONATE}

Multiple studies have reported that the therapeutic effect of itaconate involved in many diseases, which can be described from the following aspects, including anti-inflammatory, immunomodulatory, antioxidant stress, anti-bacterial, and anti-virus (Figure 3). The application of itaconate in these diseases reflects its extensive regulatory potential (Tables 2, 3).

\section{The Effect of Itaconate in Anti-inflammation Sepsis}

The definition of Sepsis 3.0 is the life-threatening organ dysfunction caused by a host's inappropriate response to infection, emphasizing the imbalance between inflammation and immune homeostasis in sepsis progression (Cecconi et al., 2018). During the early stage of infection, the monocytemacrophage system and neutrophils are continuously activated under the stimulation of pathogens, resulting in a massive release of pro-inflammatory cytokines (van der Poll et al., 2017). In an LPS-induced sepsis mice model, administration of DI or 4-OI protected mice from death and inhibited excessive inflammation. Zhang et al. showed that the administration of DI enhanced survival rate, decreased serum level of tumor necrosis factor- $\alpha$ (TNF- $\alpha$ ) and IL-6, and ameliorated lung injury in septic mice and BMDMs via promoting the expression of Nrf2 and its downstream factor Heme Oxygenase 1(HO-1) and quinoneoxidore-ductase 1(NQO-1) (Zhang et al., 2021), which was consistent with that of Mills, Evanna L (Mills et al., 2018). However, as the disease develops, the high inflammatory storm evolves into immunosuppression or immunoparalysis, which increases the susceptibility to secondary infections (Biswas and Lopez-Collazo, 2009). Irg1 was highly upregulated both in sepsis patients and mice with LPS tolerance models, which indicated that itaconate might be involved in the immunoparalysis of sepsis (Li et al., 2013). However, the continual decarboxylation of the TCA intermediate cisaconitate to itaconate resulted in a long-term, low-energy state, which may be a pathological state exacerbated immunoparalysis (Zhu et al., 2020). Zhu et al. found when reversed the increase of itaconate rejuvenated the anabolic TCA cycle, and increased the ATP produced by the mitochondrial electron transport chain thereby improved immune tolerance in septic shock model and THP-1 human monocytes during sepsis-like inflammatory responses (Zhu et al., 2020; Mainali et al., 2021). Intriguingly, monocytes treated with $\beta$-glucan can inhibit the expression of Irg1 induced by LPS and revert the potential harmful effects of itaconate (Dominguez-Andres et al., 2019). These studies have thoroughly and carefully distinguished the potential applications of itaconate in different periods of sepsis. The proper anti-inflammatory effect of itaconate during the excessive inflammation period the reduction of itaconate in the 


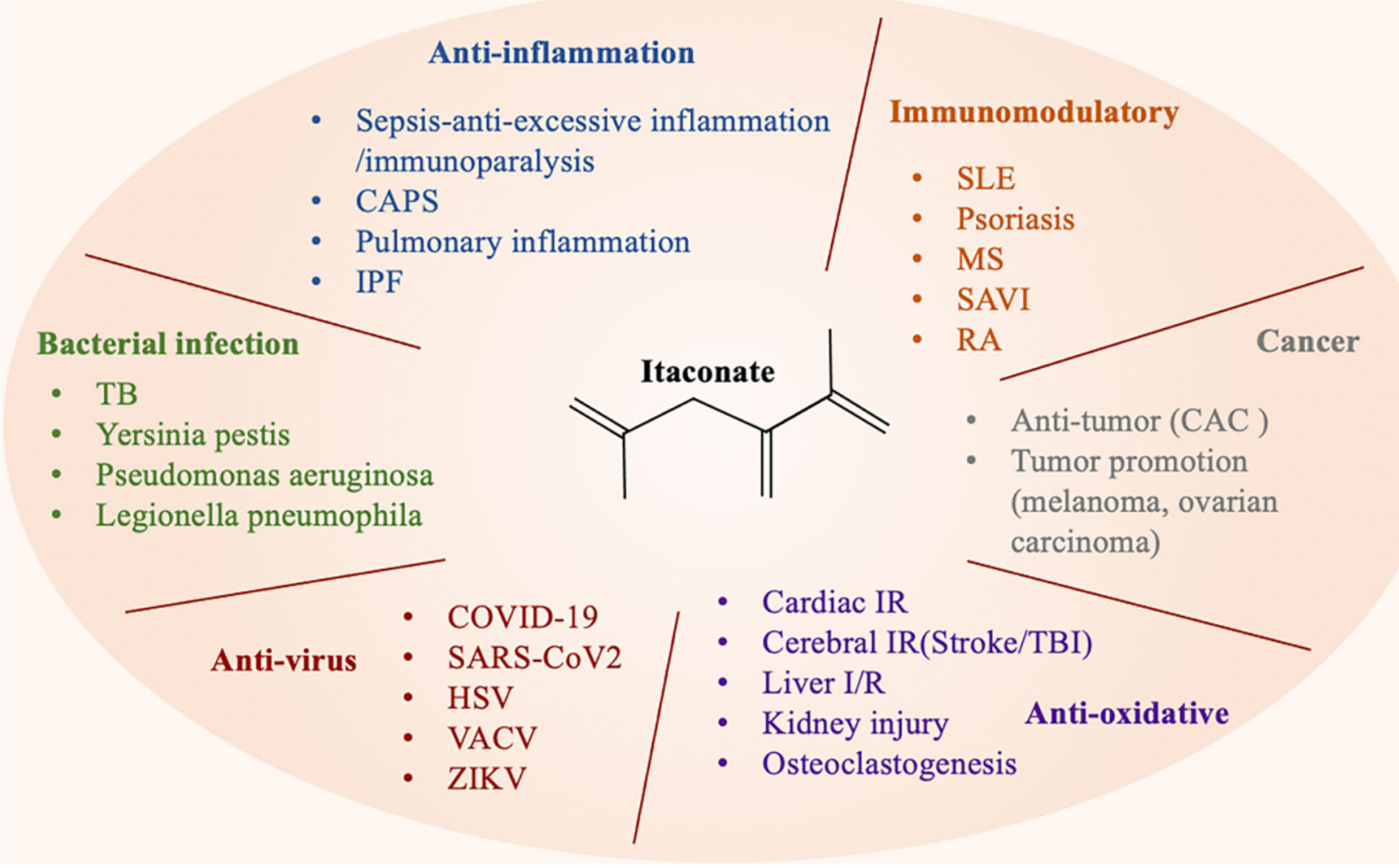

FIGURE 3 | Itaconate can be involved in various types of diseases through a variety of regulatory ways.

immunoparalysis phase can effectively control the progression of sepsis.

\section{CAPS}

Cryopyrin-associated periodic syndrome (CAPS) is an inherited autoinflammatory disease with hyperactive nod-like receptor protein 3 (NLRP3) (Ohnishi et al., 2012), an inflammasome as one of the best characterized intracellular multi-protein complexes is a critical determinant of a wide range of different autoinflammatory conditions (Swanson et al., 2019). The control of NLRP3 inflammasome activation may be a critical role in potential target of treatment (Prochnicki and Latz, 2017). A new research published on journal of Cell Metabolism found that 4-OI could be converted to intracellular itaconate by macrophage and the effect of endogenous itaconate was confirmed to specific targeting of NLRP3 (Hooftman et al., 2020). Itaconate could abolish NLRP3-NEK7 connection in a modification termed dicarboxypropylation on C548 of NLRP3. They also observed that 4-OI blocked NLRP3-dependent interleukin-1 $\beta$ (IL-1 $\beta$ ) release from PBMCs isolated from CAPS patients, while SDH inhibitor did not have the same effect. Indeed, itaconate modulated LPS-regulated genes related to inflammasome activation and function, a significant portion of prototypical NLRP3-activating conditions. The targeting of
NLRP3 by itaconate provides therapeutic potential for the treatment of NLRP3-driven disorders.

\section{Pulmonary Inflammation}

Macrophage-driven lung inflammation is associated with particulate matter (PM) air pollution (McGlade and Landrigan, 2019). Exposure to pollution particles led to the increase of mitochondrial oxygen consumption rate and ROS eventually induce the production of TNFa and IL-6 (Soberanes et al., 2019). RNA-Seq analysis showed that Acod1 gene encoding the enzyme producing itaconate was significant activated in the polluted environment exposed to pulmonary macrophages in a timedependent manner. Itaconate was indispensable for regulating its downstream antioxidant genes and inflammatory genes (Sun et al., 2020). If treated with 4-OI, the Nrf2 pathway would be opened and succinate was also accumulated which eventually reduced pulmonary inflammation. Similar results could be obtained when treatment before exposure to contamination. Itaconate was also found to alleviate idiopathic pulmonary fibrosis (IPF) through changing macrophage phenotype and function (Wynn and Vannella, 2016; Allden et al., 2019). A recent study found that the expression of ACOD1/itaconate was obviously reduced in patients with IPF, this reduction was proved to exacerbate more disease severity of pulmonary fibrosis in mice (Ogger et al., 2020). Administration or inhalation of exogenous 
TABLE 2 | The participation mechanisms of itaconate in different diseases.

\begin{tabular}{|c|c|c|c|c|c|}
\hline Diseases & & Animals and cell & Agents and methods & Main mechanisms & References \\
\hline \multirow[t]{6}{*}{ Anti-inflammation } & Sepsis & $\begin{array}{l}\text { C57(B6) mice } \\
\text { BMDMs(mouse) } \\
\text { PBMCs(human) } \\
\text { RAW264.7 macrophages }\end{array}$ & $\begin{array}{l}\text { LPS (Sigma; } 2.5 \text { mg/kg;100 ng/ml) } \\
\text { OI (Sigma, } 50 \text { mg/kg, } 125 \mu \mathrm{M})\end{array}$ & Keap1/Nrf2-IFN & Mills et al., 2018 \\
\hline & & $\begin{array}{l}\text { BMDMs(mouse) } \\
\text { C57(B/6J)mice }\end{array}$ & $\begin{array}{l}\text { LPS (Sigma, } 0.1 \mu \mathrm{g} / \mathrm{mouse} ; 100 \mathrm{ng} / \mathrm{mL} \text { ) } \\
\text { D-galactosamine }(0.5 \mathrm{mg} / \mathrm{g}, 0.5 \mathrm{mg} / \mathrm{g}) \\
\text { 4-OI (50 mg/kg; } 200 \mu \mathrm{M})\end{array}$ & Nrf2- HO-1/NQO-1 & Zhang et al., 2021 \\
\hline & & $\begin{array}{l}\text { Whole blood (sepsis patients) } \\
\text { C57(B/6J) mice } \\
\text { Primary peritoneal macrophages } \\
\text { RAW264.7 macrophages } \\
\text { THP-1 cells(human) }\end{array}$ & LPS (Sigma, $100 \mathrm{ng} / \mathrm{mL} ; 1 \mu \mathrm{g} / \mathrm{ml})$ & $\begin{array}{l}\text { Itaconate induce immunoparalysis } \\
\beta \text {-glucan reverse immunoparalysis } \\
\text { made by itaconate }\end{array}$ & $\begin{array}{l}\text { Li et al., 2013; } \\
\text { Dominguez-Andres } \\
\text { et al., 2019; Mainali } \\
\text { et al., 2021 }\end{array}$ \\
\hline & CAPS & $\begin{array}{l}\text { C57(B/6J) mice } \\
\text { PBMCs, monocytes human } \\
\text { HEK293T Cells }\end{array}$ & $\begin{array}{l}\text { LPS }(200 \mathrm{ng} / \mathrm{mL}) \\
4-\mathrm{Ol}(50 \mathrm{mg} / \mathrm{kg})\end{array}$ & NLRP3- IL-1 $\beta$ & Hooftman et al., 2020 \\
\hline & $\begin{array}{l}\text { PM-Pulmonary } \\
\text { inflammation }\end{array}$ & $\begin{array}{l}\text { C57(B/6N,6J)mice } \\
\text { BMDMs(mouse) }\end{array}$ & $\begin{array}{l}\text { LPS (Santa; } 100 \mathrm{ng} / \mathrm{ml}) \\
\text { Itaconate (sigma; } 10 \mathrm{mM}) \\
\text { 4-OI (sigma;0.25 mM) }\end{array}$ & ACOD1-SDH inhibition & Sun et al., 2020 \\
\hline & IPF & $\begin{array}{l}\text { C57(B6)mice primary AMs, HLFs(human) } \\
\text { HBEs(human) }\end{array}$ & Itaconate (Sigma, 0.25 mg/kg) & ACOD1-antifibrotic & Ogger et al., 2020 \\
\hline \multirow[t]{4}{*}{ Immunomodulatory } & SLE & $\begin{array}{l}\text { THP-1 macrophages(human) } \\
\text { PBMCs(human) }\end{array}$ & $\begin{array}{l}\text { LPS (Sigma; } 500 \mathrm{ng} / \mathrm{mL}) \\
4-\mathrm{Ol}(25 \mu \mathrm{M}, 2 \mathrm{~h})\end{array}$ & Keap1-Nrf2-NF-кB & Tang et al., 2018 \\
\hline & Psoriasis & $\begin{array}{l}\text { BMDMs(mouse) } \\
\text { C57(B6,B/6N,6J) mice } \\
\text { BV2 microglial cell }\end{array}$ & $\begin{array}{l}\text { LPS (Sigma; } 100 \mathrm{ng} / \mathrm{mL} \text { ) } \\
\text { DI (Sigma; } 20 \text { mg/mouse, } 250 \mu \mathrm{M}) \\
\text { DMF (Sigma, } 50 \mu \mathrm{M} \text { ) }\end{array}$ & DI-IkBל- IL-17 & $\begin{array}{l}\text { Bambouskova et al., } \\
2018\end{array}$ \\
\hline & Multiple sclerosis & $\begin{array}{l}\text { C57(B6),SJL/J mice } \\
\text { Microglia(mice) } \\
\text { Mononuclear cell(mice) }\end{array}$ & $\begin{array}{l}\text { LPS (Sigma; } 100 \text { ng/mL) } \\
\text { DMI (Sigma; } 400 \text { mg/kg, } 150 \mu \mathrm{M})\end{array}$ & $\begin{array}{l}\text { MMP3, MMP9 inhibition inhibite } \\
\text { Th1/Th17 differentiation and } \\
\text { infiltration to CNS }\end{array}$ & Kuo et al., 2020 \\
\hline & SAVI & $\begin{array}{l}\text { THP-1 cells, PBMCs, HaCat HEK293T, A549 } \\
\text { cells(human) }\end{array}$ & 4-OI (Aarhus University; $125 \mu \mathrm{M}, 200 \mu \mathrm{M}$ ) & Nrf2-STING-IFN & Olagnier et al., 2018 \\
\hline \multirow[t]{5}{*}{ Anti-oxidation } & Heart & $\begin{array}{l}\text { C57(B6, B/6N) mice } \\
\text { BMDM (mouse) } \\
\text { RAW264.7 macrophages }\end{array}$ & $\begin{array}{l}\text { LPS (Sigma; } 100 \mathrm{ng} / \mathrm{mL}) \\
\text { DI (4 mg/kg/min;0.25 mM) }\end{array}$ & $\begin{array}{l}\text { SDH inhibition } \\
\text { HIF- } 1 \alpha / \mathrm{IL}-1 \beta, \text { IL-18 reduction not } \\
\text { TNF- } \alpha \text { production }\end{array}$ & $\begin{array}{l}\text { Lampropoulou et al., } \\
2016\end{array}$ \\
\hline & Brain & $\begin{array}{l}\text { C57(B/6J) mice } \\
\text { Primary cortical neurons, astrocytes(SD rat) }\end{array}$ & Itaconate (15 mg/kg/min) & $\begin{array}{l}\text { SDH inhibition } \\
\text { ROS/RNS reduction }\end{array}$ & Cordes et al., 2020 \\
\hline & & C57(B6) mice & DMI (Sigma, 20 mg) & Inhibited toxic conversion of microglia & Zhang et al., 2019 \\
\hline & Liver & $\begin{array}{l}\text { C57 (B/6N, B/6J) mice } \\
\text { hepatocytes(human,mouse) } \\
\text { NPCs(mouse) }\end{array}$ & 4-OI (25 mg/kg, 62.5/125 $\mu \mathrm{M})$ & IRG1-Nrf2- antioxidant & RajanBabu et al., 1999 \\
\hline & Kidney & $\begin{array}{l}\text { SD Rat } \\
\text { HK-2 cell }\end{array}$ & 4-Ol (1, $10 \mathrm{mg} / \mathrm{kg} ; 1,10,30,100 \mu \mathrm{mol} / \mathrm{L})$ & 4-OI-TGF- $\mathrm{B} /$ Smad- NF-kB & Tang et al., 2019 \\
\hline
\end{tabular}




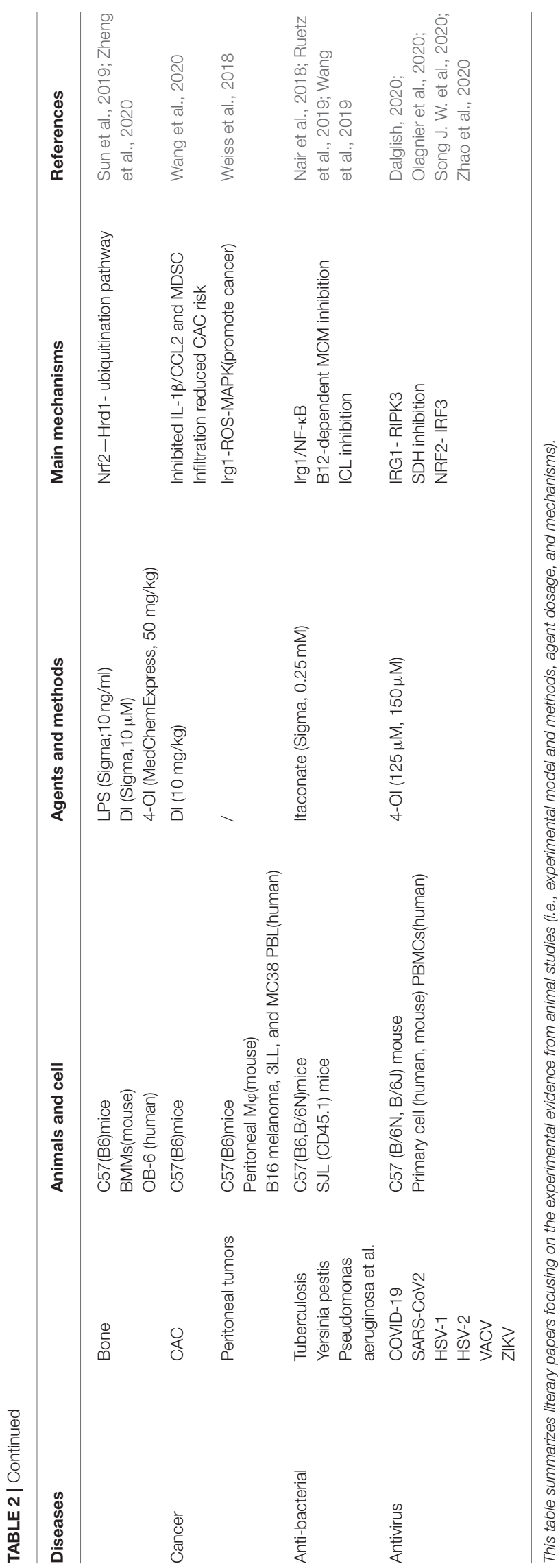

itaconate reversed the formulation of pulmonary fibrosis through changing the fibroblast metabolic phenotype, decreased proliferative capacity, and limited wound healing of human lung fibroblasts. 4-OI was also found play an extremely important role in alleviating acute lung injury (ALI) or acute respiratory distress syndrome (ARDS) by inhibiting the expression of the downstream inflammatory cytokines (IL- $\beta$, IL-6, and TNF- $\alpha$ ) and ROS to increase the activity of antioxidants in lung tissue ( $\mathrm{Li}$ et al., 2020).

\section{Abdominal Aortic Aneurysm}

Itaconate, kind of inflammation-related endogenous metabolites serve as a novel and inexpensive therapeutic target to control the progression of vascular inflammation disease -abdominal aortic aneurysm (AAA) (Song H. et al., 2020). And Irg1/itaconate pathway was involved in the formation of AAA. Exogenous itaconate addition in apolipoprotein E-deficient (Apoe ${ }^{-/-}$) mice suppressed the initiation and progression of AAA and downstream inflammatory protein while Irg1 deficiency reverted the effect of inhibitory. Overexpression of Keap1 or transferred Cys151S mutant Keap1 vector also abolished the activation of Nrf2 induced by itaconate.

\section{Mastitis/Endometritis}

In two studies explored about the protective effect of DI to prevent the pathology inflammation of mastitis/endometritis diseases (Zhao et al., 2019; Xu et al., 2020). Injection of DI markedly decreased the production of pro-inflammatory cytokines, and increased the expression of Nrf2, HO-1 in LPSinduced mastitis and endometritis mice. It also inhibited the signal pathway of TLR4 and phosphorylation of p65 nuclear factor kappa B (NF-кB). Their researches investigated that DI may serve as a potential candidate to protect against pathological damage of mastitis/endometritis.

\section{The Effect of Itaconate in Immunomodulatory Psoriasis}

Immunometabolism, as a burgeoning field has linked intracellular metabolic pathways to immune-mediated inflammation conditions (Diskin et al., 2020). Itaconate is perhaps the crucial determinant of immune-mediated diseases because of its multiple immunomodulatory and antiinflammatory effects. Psoriasis is well-known as an autoimmune disease of the joints and skin with inappropriate $\mathrm{T}$ cell activation. The transcriptional activator $\mathrm{I} \kappa \mathrm{B} \zeta$ induced by IL-17A-treated epithelial cells plays a key role in mediating the orchestrates downstream inflammatory responses in immune-related conditions, psoriasis (Bambouskova et al., 2018; Bertelsen et al., 2020). DI pretreatment interfere with the production of I $\mathrm{B} \zeta$ in a way of electrophilic stress mediated by ATF3, a key mediator of the Nrf2-independent way and downregulate $\mathrm{I} \kappa \mathrm{B} \zeta$ protein correlated genes in primary keratinocytes stimulated by IL-17A (Bambouskova et al., 2018). No significant changes of scaling or oedema of the skin was observed in a mouse model of psoriasis-like pathology condition treated with DI. And daily DI administration has a favorable safety profile with 
TABLE 3 | The role of itaconate and its potential clinical application.

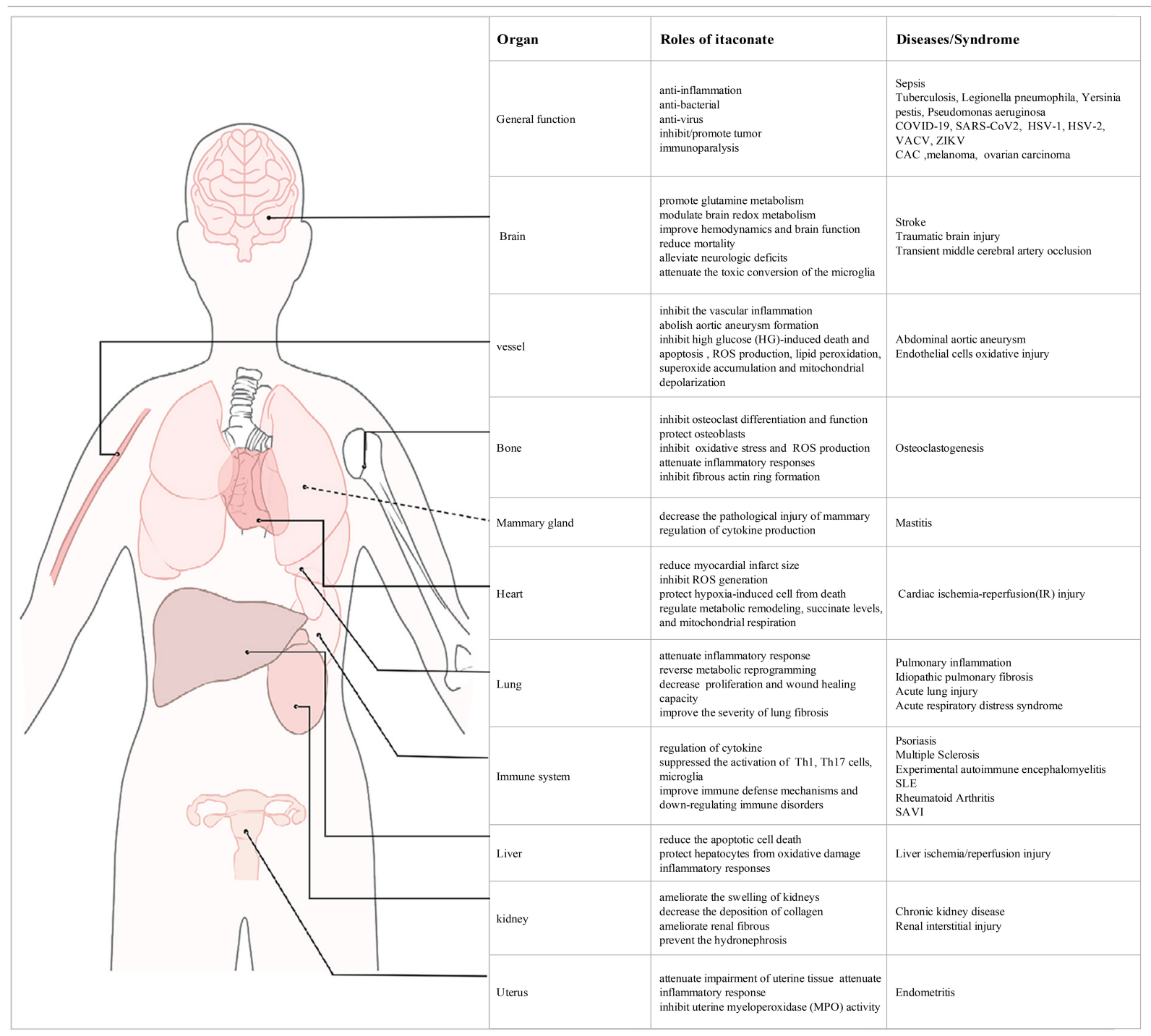

Itaconate plays multiple roles in different tissues and disease conditions and this table highlights to show the utility of itaconate for potential clinical.

little effect in the heart and the liver. Targeting the DI-I $\kappa \mathrm{B} \zeta$ regulatory axis may be a new approach to subside the symptoms of autoimmune condition.

\section{Multiple Sclerosis}

Multiple sclerosis (MS) is a progressive demyelinating destruction associated with immune-mediated pathogenesis of central nervous system (Faissner et al., 2019). A recent study showed the immunomodulatory effect of DI in an Experimental autoimmune encephalomyelitis (EAE) animal model commonly represented MS (Kuo et al., 2020). DI is capable of decreased production of MMP3 and MMP9 which lead to the lessen of BBB disruption (Brown, 2001; Mirowska-Guzel et al., 2009). DI suppressed the activation of microglia thus decreased the infiltration and differentiation of encephalitogenic Th1 and
Th17 cells in EAE through a Nrf2-independent mechanism. The multiple cellular and molecular defense pathway of DI ameliorated the severity of chronic EAE condition. Dimethyl Fumarate (DMF) is the traditional immunomodulatory drug of MS and psoriasis dependent on GAPDH blockage and aerobic glycolysis inhibition (Kornberg et al., 2018). Itaconate has the same effect on GAPDH. However, inferred from the mechanism of DMF, whether there are other mechanisms of itaconate in the treatment of MS need to be further confirmed.

\section{SLE}

Systemic lupus erythematosus (SLE) is a common condition characterized by the dysregulation of pro- and anti-inflammatory cytokines (Tsokos et al., 2016). Treatments targeted inflammatory cytokines which induces a significant clinical improvement in 
patients, such as BENLYSTA and other biological agents have been widely used (Lee et al., 2011). Tang et al. (2018) reported that TNF- $\alpha$, IL- $1 \beta$, and IL-6, were significantly decreased in the PBMCs of SLE patients and THP-1 cells treated with OI. The probably mechanism they found was that OI disassociated Keap1 from Nrf2 and exerted a potent anti-inflammatory effect by the accumulation, activation and nuclear translocation of Nrf2 protein.

\section{Rheumatoid Arthritis}

Rheumatoid arthritis (RA) is a debilitating immune-mediated disease of global prevalence (Weyand and Goronzy, 2021). The variation of plasma metabolic spectrum in patients with rheumatoid arthritis is instructive when they begin routine anti-rheumatic drug (CDMARD) treatment (Daly et al., 2020). Plasma samples from an early RA randomized strategy study (NCT00920478) were analyzed by untargeted metabolomics analysis. A total of 9 metabolites were significantly correlated with the decrease of rheumatic activity score after using CDMARD, the main components of which were itaconate and its derivatives of coenzyme A. After CDMARD (mainly methotrexate) treatment, the increase of itaconate was related to the improvement of rheumatic activity score and the decrease of C-reactive protein (CRP) level. This is consistent with its anti-inflammatory effect, which suggests that itaconate may be a sign like CRP of improvement in patients. Another recent study described itaconate as a key marker in the progress of inflammatory arthritis in Tg197 mice model, and found that the level of itaconate was increased when TNF- $\alpha$ was blocked (Michopoulos et al., 2016). Further study of itaconate pathway may reveal new important insights into the regulation of immune function and the pathogenesis of rheumatoid arthritis. It may also reveal new clinical markers of disease activity and treatment response.

\section{SAVI}

Stimulator of interferon genes (STING)-associated vasculopathy(SAVI)caused by mutation of TMEM173 gene is a system disruption of inborn innate immune disorders characterized by neonatal onset of autoinflammation diseases (Ahn and Barber, 2014). STING as an adaptor protein could induce antiviral type I IFN signaling thus signals downstream of viral (Siedel et al., 2020; Donnelly et al., 2021). Nrf2 as an important negative regulator engage on STING repression and destabilization. 4-OI that linked to Nrf2 activation was sufficient to decrease 2-fold levels of TMEM173 mRNA and the STING related-mRNA in HEK293T cells. The treatment of itaconate also inhibited the release of STING-dependent type I IFNs from SAVI-associated fibroblasts condition. Itaconate may gain a newfound foothold and valid target in the rare STING-associated inflammatory disorders (Olagnier et al., 2018).

\section{The Effect of Itaconate in Anti-oxidative} Ischemia-Reperfusion (IR) Injury

Ischemia-reperfusion(I/R) injury is a complex pathological condition which drives an imbalance of injurious metabolic processes between oxidative stress and anti-oxidant defense systems (Chamorro et al., 2016). Itaconate as a significant portion of physiological regulatory mediators participates in upregulation of succinate levels, production of inflammatory cytokine, mitochondrial respiration and directionality of the electron transport chain. A recent study demonstrated that intravenous infusion of DI induced reduction in myocardial infarct size (Lampropoulou et al., 2016) by inhibiting SDH in cardiac I/R injury on dose-dependent (Kula-Alwar et al., 2019). Pretreatment with DI exerted anti-inflammatory effects by inhibiting the expression of inducible nitric oxide synthase (iNOS) protein and secretion of IL-12p70 and IL-6 whereas no significant change of TNF- $\alpha$ was found indicating that DI-administrated probably did not affect NF- $\kappa \mathrm{B}$-dependent gene expression. DI impaired IL-1 $\beta$ production induced by not only prototypical NLRP3- dependent inflammasome activation but also absent in melanoma-2 (AIM-2)-activating conditions revealing that itaconate could exert a broader regulatory effect.

Cordes et al. found that exogenous itaconate also suppressed SDH and dramatically affected the expression levels of Hmox1, Nqo1, and Gpx1 genes, initiating the transcription of multiple antioxidant and anti-inflammatory protein in cerebral I/R injury model (Cordes et al., 2020). Zhang et al. further showed that intraperitoneal administration DI significantly alleviated neurologic deficits and promoted neural functional recovery on day 3 after surgery (Zhang et al., 2019). In addition, DI was also showed to inhibit the conversion of M1 microglia which was a critical determinant of pro-inflammatory effects. 4-OI activated Keap1-Nrf2 signaling to protect neuronal cells from hydrogen peroxide (Liu et al., 2018).

Oxidative stress is also a major contributor to liver $I / R$ injury apart from cardiac and brain Yi et al. (2020) found that IRG1-itaconate-Nrf2- antioxidant pathway protect hepatocytes from oxidative damage in liver ischemia-reperfusion and hypoxia/reoxygenation. They observed that $\operatorname{Irg} 1$ deficiency mouse rendered more susceptible to systemic and local inflammation and liver injury. Administration of 4-OI ameliorated oxidative stress and hepatocyte cell death in a manner of Nrf2-driven signaling.

\section{Diabetes Mellitus-Induced Vascular Injury}

Pathological change of vascular endothelial cell injury is the commonest cause of diabetes vasculopathy. Pre-treatment with OI was found have the protection in human umbilical vein endothelial cells (HUVECs) from high glucose (HG)-induced oxidative injury in mimicking Diabetes mellitus (Tang et al., 2019). The anti-HG protection was probably that OI abolished the association of Keap1-Nrf2 leading to the accumulation of Nrf2 translocated to cell nuclei. In consistent with other reports, this is also the reason of superior anti-oxidant activity in OI by inhibiting the production and accumulation of ROS or superoxide, decreasing the lipid peroxidation and mitochondrial depolarization. And OI nullified to against HG when Nrf2 was genetically silenced or depleted or cells transfected by Keap1 Cys151S mutation vector. 


\section{Renal Fibrosis}

Another study has found the renoprotection made by itaconate on renal fibrosis which is recognized as an inevitable pathological progression of all chronic kidney disease (CKD) (Tian et al., 2020). After OI treatment, creatinine (CRE) and blood urea nitrogen (BUN) were reduced thus prevented the occurrence of hydronephrosis. OI ameliorated renal perivascular fibrous tissue hyperplasia by the suppression the expression of transforming growth factor- $\beta$ (TGF- $\beta$ )/Smad and NF- $\kappa$ B pathways. These results also indicate the clinical application of OI through the antioxidant stress potential.

\section{Osteoclastogenesis}

4-OI affected the suppression of oxidative injury induced by hydrogen peroxide $\left(\mathrm{H}_{2} \mathrm{O}_{2}\right)$ in osteoclast-related diseases. Sun et al. found that the concentration of itaconate was lower in estrogen-deficient mice analyzed by LC-MS assay and the deficiency of Nrf2 was found to induce osteoclastogenesis (Sun et al., 2019). 4-OI could inhibit bone resorption, osteoclast differentiation and attenuated bone loss in vivo and vitro. The underlying mechanism was exogenous 4-OI modulated the ubiquitination-mediated degradation of Nrf2 thereby enhanced Nrf2 expression by suppressing the association between Nrf2 and E3 ubiquitin ligase (Hrd1) in a time-dependent manner. Furthermore, they also found that DI did not have the same effect on osteoclast function indicate that general electrophilic stress was probably not sufficient to induce the inhibition of osteoclastogenesis. In another research Zheng et al. (2020) found 4-OI protected OB- 6 cells and primary murine osteoblasts from oxidative injury, cell death, and apoptosis treated by $\mathrm{H}_{2} \mathrm{O}_{2}$ through disassociation of Keap1 and Nrf2.

\section{The Effects of Itaconate in Cancer}

Recent studies have also found that itaconate plays a vital role in cancer immunometabolism. The anti-cancer property of itaconate has been reported in colitis-associated colorectal cancer (CAC) (Wang et al., 2020). Administration of DI inhibited the secretion of cytokines IL- $1 \beta$ and chemokine ligand 2 (CCL2) in intestinal epithelial cells, reduced the infiltration of inflammatory macrophages to the tumor microenvironment (TME), thus alleviated the high inflammatory state of ulcerative colitis. The effect of itaconate was also accompanied by suppressing the cytotoxic $\mathrm{T}$ cell differentiation and their infiltration of myeloidderived suppressor cells (MDSCs), thereby reducing the risk of CAC development. In other studies, itaconate level was proved to correlate with cancer progression and the unfavorable prognosis in certain tumor types (Weiss et al., 2018). As the most crucial component in TME, tumor-associated macrophages (TAMs) can secrete various mediators such as cytokines (IL-10) and growth factors that promote tumor growth, invasion, and metastasis (Noy and Pollard, 2014; Vitale et al., 2019). The peritoneal tissue-resident macrophages $(\mathrm{pResM} \varphi)$ were found to upregulate Irg1 expression and increase itaconate level in B16 melanoma and ID8 ovarian carcinoma (Weiss et al., 2018). Itaconate was shown to increase oxidative phosphorylation (OXPHOS) with resultant production of ROS, which mediated mitogenactivated protein kinases (MAPK) activation to promote tumor progression. Conversely, when the conversion to itaconate was inhibited in $\operatorname{Irg} 1^{-/-}$mice, a significant tumor volume reduction was observed. Furthermore, itaconate promoted ROS in $\operatorname{pResM} \varphi$ through OXPHOS, which was mainly driven by the Nicotinamide adenine dinucleotide (NADH) cycle, a succinate dehydrogenaseindependent mechanism (Basit et al., 2018). Monocytes isolated from the ascetic fluid of ovarian cancer patients expressed high levels of Irg1. Similarly, another article pointed out that Irg1 played an oncogene-like role in glioma, leading to poor prognosis (Pan et al., 2014). Further research is needed to fully identify the regulatory effects of itaconate on different types of tumors.

\section{The Effects of Itaconate in Bactericidal Action}

Tuberculosis (TB) caused by Mycobacterium tuberculosis (Mtb) is the severe bacterial disease in terms of its high mortality and prevalence worldwide (Shin et al., 2011). In 2011, Shin et al. found that itaconate was increased only in Mtb-infected lung tissue for the first time by using metabolic profiles. However, the reason itaconate increased after an aerobic infection did not clarify. Nair et al. found a role for the IRG1-itaconate axis in Mtb-infected mice. Cell-intrinsic expression of Irg1 in alveolar macrophages and some dendritic cell subsets is dispensable for neutrophil recruitment and inflammation regulation. They next observed that itaconate was dramatically decreased in $\operatorname{Irg} 1^{-/-}$mice. Analogously, addition of exogenous itaconate treatment reverses the induction of inflammatory by modulating chemoattractant genes downstream of NF- $\kappa \mathrm{B}$ signaling in a $\left(\operatorname{Irg} 1^{-/-}\right) \mathrm{Mtb}-$ infected mice. These results suggested that itaconate and Irg1 played a critical role as an endogenous antibacterial effector molecule on Mtb growth (Nair et al., 2018). Another study found that the bactericidal effects of itaconate was probably due to its inhibition of B12-dependent methyl malonyl-CoA mutase (MCM) which is needed for Mtb growth on propionate as the sole carbon source. what's more, itaconate can be acylated to itaconyl-CoA (I-CoA) via a C5-dicarboxylate pathway (Ruetz et al., 2019). I-CoA also derailed the activation and repairment of Mtb MCM and the radical suicide inactivation mechanism of I-CoA was forming a markedly air-stable biradical binding with MCM (Wang et al., 2019). Moreover, a study showed that itaconate modified the proteome of Legionella pneumophila containing vacuoles to restrict intracellular bacterial pathogens (Naujoks et al., 2016). In addition, itaconate was found to restrict bacterial growth in culture by inhibiting isocitrate lyase (ICL) (a key enzyme that supports bacterial growth during infection (McFadden and Purohit, 1977). Meanwhile, the inhibitory effect of itaconate was also found in bacteria that did not express ICL enzyme, which may be due to the inhibition of acetic acid assimilation by inhibiting propionyl-CoA carboxylase (Yang et al., 2020). Some bacteria such as Yersinia pestis and Pseudomonas aeruginosa which carried genes encoding enzymes of itaconate degradation can promote pathogenicity and survival 
(Rao and McFadden, 1965; Rittenhouse and McFadden, 1974; Chen et al., 2020; Riquelme et al., 2020). Activated macrophages have been shown to produce itaconate, suggesting that these immune cells may employ this metabolite as a weapon against invading bacteria. Itaconate can exhibit bactericidal effects under acidic conditions similar to the $\mathrm{pH}$ of a macrophage phagosome (Hersch and Navarre, 2020). These studies showed that itaconate has been proposed to have direct bacteriostatic activity (Williams and O'Neill, 2018). It also implied that there was a complex evolutionary relationship between itaconate and bacteria.

\section{The Effect of Itaconate in Antivirus}

Mounting evidence discovered that an unexpected intersection between itaconate and immune activation is intricately linked with antivirus strategies. Zika virus (ZIKV), an emerging human pathogenic virus can cause significant neurologic injury by access the central nervous system (CNS) and has become an increasingly global public health challenge (Zhao et al., 2020). The production of itaconate as a direct downstream effector of ZBP1- and RIPK- IRG1 dependent transcriptional program could ameliorate viral pathogenesis in the CNS. The underlying mechanisms was probably that IRG1, a potential antiviral gene restricted replication and neuronal infection of ZIKV in neurons in a cell-intrinsic manner. Besides itaconate produced by IRG1 could alter neuronal metabolism by inhibiting the activation of SDH and a cellular environment is thereby to exert a global suppression on viral replication.

As every knows, the coronavirus disease 2019 (COVID19) has rapidly posed an unprecedented global pandemic with high morbidity, mortality, social disruption, and economic instability. But there are limited options on the prevention and treatment of this global health emergency (Dalglish, 2020). Song J. W. et al. (2020) first found there were significant difference of metabolites related to COVID-19 patients investigated in logistic regression models. Compared serum lipidome and metabolome with different stages of COVID-19 patients and healthy crowds revealed that itaconate was declining progressively with the severity of COVID-19. Works by Olagnier et al. analyzed the differentially expressed genes transcriptome between lung biopsies from COVID-19 patients by publicly available transcriptome data at first (Olagnier et al., 2020). They discovered that the expression of antioxidant genes driven by Nrf2 were significantly suppressed in COVID19 patients. 4-OI and DMF as Nrf2 inducers significantly decreased the release of progeny virus particles and the level of virus RNA in different cells infected with SARS-CoV-2. The same antiviral phenomenon treated by 4-OI occurred in other human pathogenic viruses except for vesicular stomatitis virus (VSV). 4-OI retained the antiviral replication capacity, whereas the antiviral mode of action probably not rely on the classical IFN way due to its interruption of interferon regulatory factor 3 (IRF3) activation and dimerization. These data pointed out that SARS-CoV2 targeted the Nrf2 antioxidant pathway and 4-OI as a Nrf2 inducer could perhaps be a rapidly applicable antivirus.

\section{PERSPECTIVE AND CONCLUSION}

Since the key metabolic regulation of itaconate in macrophages was revealed, people have begun to recognize the complex interaction between metabolism, immunity, and inflammation, which provides us a new perspective for the treatment of immune inflammation-related diseases (Kabat and Pearce, 2017; Martínez-Reyes and Chandel, 2020). The signal pathways of itaconate have been summarized on Figure 2. However, in the research of Wang et al. we found that more targets of itaconate related to signal transduction, apoptosis and other signal pathways have not been discussed, and more studies are needed to supplement the mechanism of itaconate (Qin et al., 2020). Therapeutic value of itaconate in a variety of disease models (inflammation, immunomodulatory, antibacterial and antiviral, etc) can extend itaconate to the future clinical application. For example, itaconate has similar characteristics to other Nrf2 activators, while some Nrf2 agonists dimethyl fumarate have been proved to be effective in the treatment of some inflammatory diseases and used in the clinical treatment of multiple sclerosis (Kornberg et al., 2018; Carlstrom et al., 2019). This also suggests that itaconate is a very promising target for the treatment of diseases, but the current disease models are still not comprehensive enough. Further expansion of the types and models used in these studies will benefit the field of itaconate biology. Itaconate as an endogenous metabolite, will be a promising therapeutic in clinical treatment because of its low toxicity. However, most of the current therapeutic potential results come from animal models or in vitro studies. Moreover, we still need to consider that excessive immunosuppression will lead to immune paralysis and reduce the body's resistance to external infection. Although there are very few studies on itaconate in cancer, we cannot ignore its effect of tumors growth, and the progress of anti-tumors still needs more researches to support. The effect of itaconate on anti-inflammation is just like the discovery of the role of microbial metabolites as antibiotics in the 20th century may lead to the opening of the anti-inflammatory treasure house of metabolites in nature. It would be a great breakthrough to design more therapeutic itaconate derivatives to mimic the treatment of itaconate in vivo, but more analysis is needed at the beginning of clinical trials or the further structural based drug design to produce a better effect.

\section{AUTHOR CONTRIBUTIONS}

$\mathrm{JL}$ and JR designed the main ideas and wrote the article. YD was responsible for literature collection. DG was mainly responsible for language refinement and picture drawing. LY guided the whole process. All authors contributed to the article and approved the submitted version.

\section{FUNDING}

This research was supported by Zhejiang Provincial Natural Science Foundation of China under grant No. LY18H090006. 


\section{REFERENCES}

Ackermann, W. W., and Potter, V. R. (1949). Enzyme inhibition in relation to chemotherapy. Proc. Soc. Exp. Biol. Med. 72, 1-9. doi: 10.3181/00379727-72-17313

Ahn, J., and Barber, G. N. (2014). Self-DNA, STING-dependent signaling and the origins of autoinflammatory disease. Curr. Opin. Immunol. 31, 121-126. doi: 10.1016/j.coi.2014.10.009

Allden, S. J., Ogger, P. P., Ghai, P., McErlean, P., Hewitt, R., Toshner, R., et al. (2019). The transferrin receptor CD71 delineates functionally distinct airway macrophage subsets during idiopathic pulmonary fibrosis. Am. J. Respir. Crit. Care Med. 200, 209-219. doi: 10.1164/rccm.201809-1 $7750 \mathrm{OC}$

Bambouskova, M., Gorvel, L., Lampropoulou, V., Sergushichev, A., Loginicheva, E., Johnson, K., et al. (2018). Electrophilic properties of itaconate and derivatives regulate the IkappaBzeta-ATF3 inflammatory axis. Nature 556, 501-504. doi: 10.1038/s41586-018-0052-z

Basit, F., Mathan, T., Sancho, D., and de Vries, I. J. M. (2018). Human dendritic cell subsets undergo distinct metabolic reprogramming for immune response. Front. Immunol. 9:2489. doi: 10.3389/fimmu.2018.02489

Baup, S. (1836). Ueber eine neue Brenz - Citronensäure und über die Nomenclatur der Brenzkörper überhaupt. J. für Praktische Chem. 8, 418-424. doi: $10.1002 /$ prac. 18360080162

Bertelsen, T., Ljungberg, C., Litman, T., Huppertz, C., Hennze, R., Ronholt, K., et al. (2020). IkappaBzeta is a key player in the antipsoriatic effects of secukinumab. J. Allergy Clin. Immunol. 145, 379-390. doi: 10.1016/j.jaci.2019.09.029

Bezawork-Geleta, A., Rohlena, J., Dong, L., Pacak, K., and Neuzil, J. (2017). Mitochondrial complex II: at the crossroads. Trends Biochem. Sci. 42, 312-325. doi: 10.1016/j.tibs.2017.01.003

Biswas, S. K., and Lopez-Collazo, E. (2009). Endotoxin tolerance: new mechanisms, molecules and clinical significance. Trends Immunol. 30, 475-487. doi: 10.1016/j.it.2009.07.009

Blatnik, M., Frizzell, N., Thorpe, S. R., and Baynes, J. W. (2008). Inactivation of glyceraldehyde-3-phosphate dehydrogenase by fumarate in diabetes: formation of S-(2-succinyl)cysteine, a novel chemical modification of protein and possible biomarker of mitochondrial stress. Diabetes 57, 41-49. doi: 10.2337/db0 7-0838

Brown, K. A. (2001). Factors modifying the migration of lymphocytes across the blood-brain barrier. Int. Immunopharmacol. 1, 2043-2062. doi: 10.1016/S1567-5769(01)00129-1

Carlstrom, K. E., Ewing, E., Granqvist, M., Gyllenberg, A., Aeinehband, S., Enoksson, S. L., et al. (2019). Therapeutic efficacy of dimethyl fumarate in relapsing-remitting multiple sclerosis associates with ROS pathway in monocytes. Nat. Commun. 10:3081. doi: 10.1038/s41467-019-1 1139-3

Cecconi, M., Evans, L., Levy, M., and Rhodes, A. (2018). Sepsis and septic shock. Lancet 392, 75-87. doi: 10.1016/S0140-6736(18)30696-2

Chamorro, A., Dirnagl, U., Urra, X., and Planas, A. M. (2016). Neuroprotection in acute stroke: targeting excitotoxicity, oxidative and nitrosative stress, and inflammation. Lancet Neurol. 15, 869-881. doi: 10.1016/S1474-4422(16)00114-9

Chen, M., Sun, H., Boot, M., Shao, L., Chang, S. J., Wang, W., et al. (2020). Itaconate is an effector of a Rab GTPase cell-autonomous host defense pathway against Salmonella. Science 369, 450-455. doi: 10.1126/science.aaz1333

Cordes, T., Lucas, A., Divakaruni, A. S., Murphy, A. N., Cabrales, P., and Metallo, C. M. (2020). Itaconate modulates tricarboxylic acid and redox metabolism to mitigate reperfusion injury. Mol. Metab. 32, 122-135. doi: 10.1016/j.molmet.2019.11.019

Cordes, T., and Metallo, C. M. (2021). Itaconate Alters Succinate and Coenzyme A Metabolism via Inhibition of Mitochondrial Complex II and Methylmalonyl-CoA Mutase. Metabolites 11:117. doi: 10.3390/metabo110 20117

Cordes, T., Wallace, M., Michelucci, A., Divakaruni, A. S., Sapcariu, S. C., Sousa, C., et al. (2016). Immunoresponsive Gene 1 and itaconate inhibit succinate dehydrogenase to modulate intracellular succinate levels. J. Biol. Chem. 291, 14274-14284. doi: 10.1074/jbc.M115.685792
Cuadrado, A., Rojo, A. I., Wells, G., Hayes, J. D., Cousin, S. P., Rumsey, W. L., et al. (2019). Therapeutic targeting of the NRF2 and KEAP1 partnership in chronic diseases. Nat. Rev. Drug Discov. 18, 295-317. doi: 10.1038/s41573-018-0008-x

Dalglish, S. L. (2020). COVID-19 gives the lie to global health expertise. Lancet 395:1189. doi: 10.1016/S0140-6736(20)30739-X

Daly, R., Blackburn, G., Best, C., Goodyear, C. S., Mudaliar, M., Burgess, K., et al. (2020). Changes in plasma itaconate elevation in early rheumatoid arthritis patients elucidates disease activity associated macrophage activation. Metabolites 10:241. doi: 10.3390/metabo10060241

Denko, N. C. (2008). Hypoxia, HIF1 and glucose metabolism in the solid tumour. Nat. Rev. Cancer 8, 705-713. doi: 10.1038/nrc2468

Diskin, C., Ryan, T. A. J., and O'Neill, L. A. J. (2020). Modification of proteins by metabolites in immunity. Immunity 54, 19-31. doi: 10.1016/j.immuni.2020.09.014

Dominguez-Andres, J., Novakovic, B., Li, Y., Scicluna, B. P., Gresnigt, M. S., Arts, R. J. W., et al. (2019). The itaconate pathway is a central regulatory node linking innate immune tolerance and trained immunity. Cell Metab. 29, 211-220 e215. doi: 10.1016/j.cmet.2018.09.003

Donnelly, C. R., Jiang, C., Andriessen, A. S., Wang, K., Wang, Z., Ding, H., et al. (2021). STING controls nociception via type I interferon signalling in sensory neurons. Nature 591, 275-280. doi: 10.1038/s41586-020-03151-1

ElAzzouny, M., Tom, C. T., Evans, C. R., Olson, L. L., Tanga, M. J., Gallagher, K. A., et al. (2017). Dimethyl itaconate is not metabolized into itaconate intracellularly. J. Biol. Chem. 292, 4766-4769. doi: 10.1074/jbc.C117.775270

Faissner, S., Plemel, J. R., Gold, R., and Yong, V. W. (2019). Progressive multiple sclerosis: from pathophysiology to therapeutic strategies. Nat. Rev. Drug Discov. 18, 905-922. doi: 10.1038/s41573-019-0035-2

Ghosh, S., and Hayden, M. S. (2008). New regulators of NF-kappaB in inflammation. Nat. Rev. Immunol. 8, 837-848. doi: 10.1038/nri2423

Hersch, S. J., and Navarre, W. W. (2020). The salmonella LysR family regulator RipR activates the SPI-13-encoded itaconate degradation cluster. Infect. Immun. 88:e00303-20. doi: 10.1128/IAI.00303-20

Hooftman, A., Angiari, S., Hester, S., Corcoran, S. E., Runtsch, M. C., Ling, C., et al. (2020). The immunomodulatory metabolite itaconate modifies nlrp3 and inhibits inflammasome activation. Cell Metab. 32, 468-478 e467. doi: 10.1016/j.cmet.2020.07.016

Hooftman, A., and O'Neill, L. A. J. (2019). The immunomodulatory potential of the metabolite itaconate. Trends Immunol. 40, 687-698. doi: 10.1016/j.it.2019.05.007

Huang, S., Braun, H. P., Gawryluk, R. M. R., and Millar, A. H. (2019). Mitochondrial complex II of plants: subunit composition, assembly, and function in respiration and signaling. Plant. J. 98, 405-417. doi: $10.1111 /$ tpj.14227

Kabat, A. M., and Pearce, E. J. (2017). Inflammation by way of macrophage metabolism. Science 356, 488-489. doi: 10.1126/science.aan2691

Kornberg, M. D., Bhargava, P., Kim, P. M., Putluri, V., Snowman, A. M., Putluri, N., et al. (2018). Dimethyl fumarate targets GAPDH and aerobic glycolysis to modulate immunity. Science 360, 449-453. doi: 10.1126/science.aan4665

Kula-Alwar, D., Prag, H. A., and Krieg, T. (2019). Targeting succinate metabolism in ischemia/reperfusion injury. Circulation 140, 1968-1970. doi: 10.1161/CIRCULATIONAHA.119.042791

Kuo, P. C., Weng, W. T., Scofield, B. A., Paraiso, H. C., Brown, D. A., Wang, P. Y., et al. (2020). Dimethyl itaconate, an itaconate derivative, exhibits immunomodulatory effects on neuroinflammation in experimental autoimmune encephalomyelitis. J. Neuroinflam. 17:138. doi: 10.1186/s12974-020-01768-7

Lampropoulou, V., Sergushichev, A., Bambouskova, M., Nair, S., Vincent, E. E., Loginicheva, E., et al. (2016). Itaconate links inhibition of succinate dehydrogenase with macrophage metabolic remodeling and regulation of inflammation. Cell Metab. 24, 158-166. doi: 10.1016/j.cmet.2016. 06.004

Lee, C. G., Jenkins, N. A., Gilbert, D. J., Copeland, N. G., and O’Brien, W. E. (1995). Cloning and analysis of gene regulation of a novel LPS-inducible cDNA. Immunogenetics 41, 263-270. doi: 10.1007/BF00172150

Lee, S. J., Silverman, E., and Bargman, J. M. (2011). The role of antimalarial agents in the treatment of SLE and lupus nephritis. Nat. Rev. Nephrol. 7, 718-729. doi: 10.1038/nrneph.2011.150 
Li, Y., Chen, X., Zhang, H., Xiao, J., Yang, C., Chen, W., et al. (2020). 4Octyl Itaconate alleviates lipopolysaccharide-induced acute lung injury in mice by inhibiting oxidative stress and inflammation. Drug Des. Devel. Ther. 14, 5547-5558. doi: 10.2147/DDDT.S280922

Li, Y., Zhang, P., Wang, C., Han, C., Meng, J., Liu, X., et al. (2013). Immune responsive gene 1 (IRG1) promotes endotoxin tolerance by increasing A20 expression in macrophages through reactive oxygen species. J. Biol. Chem. 288, 16225-16234. doi: 10.1074/jbc.M113.454538

Liao, S. T., Han, C., Xu, D. Q., Fu, X. W., Wang, J. S., and Kong, L. Y. (2019). 4-Octyl itaconate inhibits aerobic glycolysis by targeting GAPDH to exert anti-inflammatory effects. Nat. Commun. 10:5091. doi: 10.1038/s41467-019-1 3078-5

Liu, H., Feng, Y., Xu, M., Yang, J., Wang, Z., and Di, G. (2018). Fouroctyl itaconate activates Keap1-Nrf2 signaling to protect neuronal cells from hydrogen peroxide. Cell Commun. Signal. 16:81. doi: 10.1186/s12964-0180294-2

Mainali, R., Zabalawi, M., Long, D., Buechler, N., Quillen, E., Key, C. C., et al. (2021). Dichloroacetate reverses sepsis-induced hepatic metabolic dysfunction. Elife 10:e64611. doi: 10.7554/eLife.64611.sa2

Martínez-Reyes, I., and Chandel, N. (2020). Mitochondrial TCA cycle metabolites control physiology and disease. Nat. Commun. 11:102. doi: 10.1038/s41467-019-13668-3

McFadden, B. A., and Purohit, S. (1977). Itaconate, an isocitrate lyasedirected inhibitor in Pseudomonas indigofera. J. Bacteriol. 131, 136-144. doi: 10.1128/JB.131.1.136-144.1977

McGlade, J., and Landrigan, P. J. (2019). Five national academies call for global compact on air pollution and health. Lancet 394, 23. doi: 10.1016/S0140-6736(19)31417-5

Meiser, J., Kramer, L., Sapcariu, S. C., Battello, N., Ghelfi, J., D’Herouel, A. F., et al. (2016). Pro-inflammatory macrophages sustain pyruvate oxidation through pyruvate dehydrogenase for the synthesis of itaconate and to enable cytokine expression. J. Biol. Chem. 291, 3932-3946. doi: 10.1074/jbc.M115.6 76817

Michelucci, A., Cordes, T., Ghelfi, J., Pailot, A., Reiling, N., Goldmann, O., et al. (2013). Immune-responsive gene 1 protein links metabolism to immunity by catalyzing itaconic acid production. Proc. Natl. Acad. Sci. U.S.A. 110, 7820-7825. doi: 10.1073/pnas.1218599110

Michopoulos, F., Karagianni, N., Whalley, N. M., Firth, M. A., Nikolaou, C., Wilson, I. D., et al. (2016). Targeted metabolic profiling of the Tg197 mouse model reveals itaconic acid as a marker of rheumatoid arthritis. J. Proteome Res. 15, 4579-4590. doi: 10.1021/acs.jproteome.6b00654

Mills, E. L., Kelly, B., Logan, A., Costa, A. S. H., Varma, M., Bryant, C. E., et al. (2016). Succinate dehydrogenase supports metabolic repurposing of mitochondria to drive inflammatory macrophages. Cell 167, 457-470 e413. doi: 10.1016/j.cell.2016.08.064

Mills, E. L., Ryan, D. G., Prag, H. A., Dikovskaya, D., Menon, D., Zaslona, Z., et al. (2018). Itaconate is an anti-inflammatory metabolite that activates Nrf2 via alkylation of KEAP1. Nature 556, 113-117. doi: 10.1038/nature 25986

Mirowska-Guzel, D., Gromadzka, G., Czlonkowski, A., and Czlonkowska, A. (2009). Association of MMP1, MMP3, MMP9, and MMP12 polymorphisms with risk and clinical course of multiple sclerosis in a Polish population. J. Neuroimmunol. 214, 113-117. doi: 10.1016/j.jneuroim.2009.06.014

Murphy, M., and O'Neill, L. (2018). Krebs cycle reimagined: the emerging roles of succinate and itaconate as signal transducers. Cell 174, 780-784. doi: 10.1016/j.cell.2018.07.030

Nair, S., Huynh, J. P., Lampropoulou, V., Loginicheva, E., Esaulova, E., Gounder, A. P., et al. (2018). Irg1 expression in myeloid cells prevents immunopathology during M. tuberculosis infection. J. Exp. Med. 215, 1035-1045. doi: 10.1084/jem.20180118

Naujoks, J., Tabeling, C., Dill, B. D., Hoffmann, C., Brown, A. S., Kunze, M., et al. (2016). IFNs modify the proteome of legionella-containing vacuoles and restrict infection Via IRG1-derived itaconic acid. PLoS Pathog. 12:e1005408. doi: 10.1371/journal.ppat.1005408

Nonnenmacher, Y., and Hiller, K. (2018). Biochemistry of proinflammatory macrophage activation. Cell Mol. Life Sci. 75, 2093-2109. doi: $10.1007 / \mathrm{s} 00018-018-2784-1$
Noy, R., and Pollard, J. W. (2014). Tumor-associated macrophages: from mechanisms to therapy. Immunity 41, 49-61. doi: 10.1016/j.immuni.2014.06.010

Ogger, P. P., Albers, G. J., Hewitt, R. J., O’Sullivan, B. J., Powell, J. E., Calamita, E., et al. (2020). Itaconate controls the severity of pulmonary fibrosis. Sci. Immunol. 5:eabc1884. doi: 10.1126/sciimmunol.abc1884

Ohnishi, H., Teramoto, T., Iwata, H., Kato, Z., Kimura, T., Kubota, K., et al. (2012). Characterization of NLRP3 variants in Japanese cryopyrinassociated periodic syndrome patients. J. Clin. Immunol. 32, 221-229. doi: 10.1007/s10875-011-9629-0

Olagnier, D., Brandtoft, A. M., Gunderstofte, C., Villadsen, N. L., Krapp, C. Thielke, A. L., et al. (2018). Nrf2 negatively regulates STING indicating a link between antiviral sensing and metabolic reprogramming. Nat. Commun. 9:3506. doi: 10.1038/s41467-018-05861-7

Olagnier, D., Farahani, E., Thyrsted, J., Blay-Cadanet, J., Herengt, A., Idorn, M., et al. (2020). SARS-CoV2-mediated suppression of NRF2-signaling reveals potent antiviral and anti-inflammatory activity of 4-octyl-itaconate and dimethyl fumarate. Nat. Commun. 11:4938. doi: 10.1038/s41467-020-1 8764-3

O'Neill, L. A. J., and Artyomov, M. N. (2019). Itaconate: the poster child of metabolic reprogramming in macrophage function. Nat. Rev. Immunol. 19, 273-281. doi: 10.1038/s41577-019-0128-5

Pan, J., Zhao, X., Lin, C., Xu, H., Yin, Z., Liu, T., et al. (2014). Immune responsive gene 1, a novel oncogene, increases the growth and tumorigenicity of glioma. Oncol. Rep. 32, 1957-1966. doi: 10.3892/or.2014.3474

Prochnicki, T., and Latz, E. (2017). Inflammasomes on the crossroads of innate immune recognition and metabolic control. Cell Metab. 26, 71-93. doi: 10.1016/j.cmet.2017.06.018

Qin, W., Qin, K., Zhang, Y., Jia, W., Chen, Y., Cheng, B., et al. (2019). S-glycosylation-based cysteine profiling reveals regulation of glycolysis by itaconate. Nat. Chem. Biol. 15, 983-991. doi: 10.1038/s41589-0190323-5

Qin, W., Zhang, Y., Tang, H., Liu, D., Chen, Y., Liu, Y., et al. (2020). Chemoproteomic profiling of itaconation by bioorthogonal probes in inflammatory macrophages. J. Am. Chem. Soc. 142, 10894-10898. doi: $10.1021 /$ jacs.9b11962

RajanBabu, T. V., Radetich, B., You, K. K., Ayers, T. A., Casalnuovo, A. L., and Calabrese, J. C. (1999). Electronic effects in asymmetric catalysis: structural studies of precatalysts and intermediates in Rh-Catalyzed hydrogenation of dimethyl itaconate and acetamidocinnamic acid derivatives using C(2)-symmetric diarylphosphinite ligands. J. Org. Chem. 64, 3429-3447. doi: $10.1021 /$ jo9901182

Rao, D., Hussain, M., Rangadu, V., K, S., Krishna, G., and Swamy, A. (2006). Fermentatative production of itaconic acid by Aspergillus terreus using Jatropha seed cake. African J. Biotechno. 6, 2140-2142. doi: 10.5897/AJB2007.000-2333

Rao, G. R., and McFadden, B. A. (1965). Isocitrate lyase from Pseudomonas indigofera. IV. specificity and inhibition. Arch. Biochem. Biophys. 112, 294-303. doi: 10.1016/0003-9861(65)90049-4

Riquelme, S. A., Liimatta, K., Wong Fok Lung, T., Fields, B., Ahn, D., Chen, D., et al. (2020). Pseudomonas aeruginosa Utilizes HostDerived Itaconate to Redirect Its Metabolism to Promote Biofilm Formation. Cell Metab. 31, 1091-1106 e1096. doi: 10.1016/j.cmet.2020. 04.017

Rittenhouse, J. W., and McFadden, B. A. (1974). Inhibition of isocitrate lyase from Pseudomonas indigofera by itaconate. Arch. Biochem. Biophys. 163, 79-86. doi: 10.1016/0003-9861(74)90456-1

Robert, T., and Friebel, S. (2016). Itaconic acid - a versatile building block for renewable polyesters with enhanced functionality. Green Chem. 8, 2922-2934 doi: 10.1039/C6GC00605A

Ruetz, M., Campanello, G. C., Purchal, M., Shen, H., McDevitt, L., Gouda, H., et al. (2019). Itaconyl-CoA forms a stable biradical in methylmalonylCoA mutase and derails its activity and repair. Science 366, 589-593. doi: 10.1126/science.aay0934

Russell, D. G., Huang, L., and VanderVen, B. C. (2019). Immunometabolism at the interface between macrophages and pathogens. Nat. Rev. Immunol. 19, 291-304. doi: 10.1038/s41577-019-0124-9 
Ryan, D. G., Murphy, M. P., Frezza, C., Prag, H. A., Chouchani, E. T., O’Neill, L. A., et al. (2019). Coupling Krebs cycle metabolites to signalling in immunity and cancer. Nat. Metab. 1, 16-33. doi: 10.1038/s42255-018-0014-7

Sano, M., Tanaka, T., Ohara, H., and Aso, Y. (2020). Itaconic acid derivatives: structure, function, biosynthesis, and perspectives. Appl. Microbiol. Biotechnol. 104, 9041-9051. doi: 10.1007/s00253-020-10908-1

Schmidt, T., Dai, Z., Drexler, H. J., Baumann, W., Jager, C., Pfeifer, D., et al. (2008). Novel contributions to the mechanism of the enantioselective hydrogenation of dimethyl itaconate with rhodium complexes. Chemistry 14, 4469-4471. doi: $10.1002 /$ chem. 200800389

Shin, J. H., Yang, J. Y., Jeon, B. Y., Yoon, Y. J., Cho, S. N., Kang, Y. H., et al. (2011). (1)H NMR-based metabolomic profiling in mice infected with Mycobacterium tuberculosis. J. Proteome Res. 10, 2238-2247. doi: 10.1021/pr1 $01054 \mathrm{~m}$

Siedel, H., Roers, A., Rosen-Wolff, A., and Luksch, H. (2020). Type I interferonindependent $\mathrm{T}$ cell impairment in a Tmem173 N153S/WT mouse model of STING associated vasculopathy with onset in infancy (SAVI). Clin. Immunol. 216:108466. doi: 10.1016/j.clim.2020.108466

Soberanes, S., Misharin, A. V., Jairaman, A., Morales-Nebreda, L., McQuattiePimentel, A. C., Cho, T., et al. (2019). Metformin targets mitochondrial electron transport to reduce air-pollution-induced thrombosis. Cell Metab. 29, 503. doi: 10.1016/j.cmet.2018.12.001

Song, H., Xu, T., Feng, X., Lai, Y., Yang, Y., Zheng, H., et al. (2020). Itaconate prevents abdominal aortic aneurysm formation through inhibiting inflammation via activation of Nrf2. EBioMedicine 57:102832. doi: 10.1016/j.ebiom.2020.102832

Song, J. W., Lam, S. M., Fan, X., Cao, W. J., Wang, S. Y., Tian, H., et al. (2020). Omics-driven systems interrogation of metabolic dysregulation in COVID19 pathogenesis. Cell Metab. 32, 188-202 e185. doi: 10.1016/j.cmet.2020. 06.016

Sun, K. A., Li, Y., Meliton, A. Y., Woods, P. S., Kimmig, L. M., CetinAtalay, R., et al. (2020). Endogenous itaconate is not required for particulate matter-induced NRF2 expression or inflammatory response. Elife 9:e54877. doi: 10.7554/eLife.54877.sa2

Sun, X., Zhang, B., Pan, X., Huang, H., Xie, Z., Ma, Y., et al. (2019). Octyl itaconate inhibits osteoclastogenesis by suppressing $\mathrm{Hrdl}$ and activating Nrf2 signaling. FASEB J. 33, 12929-12940. doi: 10.1096/fj.201900 887RR

Swain, A., Bambouskova, M., Kim, H., Andhey, P. S., Duncan, D., Auclair, K., et al. (2020). Comparative evaluation of itaconate and its derivatives reveals divergent inflammasome and type I interferon regulation in macrophages. Nat. Metab. 2, 594-602. doi: 10.1038/s42255-020-0210-0

Swanson, K. V., Deng, M., and Ting, J. P. (2019). The NLRP3 inflammasome: molecular activation and regulation to therapeutics. Nat. Rev. Immunol. 19, 477-489. doi: 10.1038/s41577-019-0165-0

Tang, C., Tan, S., Zhang, Y., Dong, L., and Xu, Y. (2019). Activation of Keap1Nrf2 signaling by 4-octyl itaconate protects human umbilical vein endothelial cells from high glucose. Biochem. Biophys. Res. Commun. 508, 921-927. doi: 10.1016/j.bbrc.2018.12.032

Tang, C., Wang, X., Xie, Y., Cai, X., Yu, N., Hu, Y., et al. (2018). 4-Octyl Itaconate Activates Nrf2 signaling to inhibit pro-inflammatory cytokine production in peripheral blood mononuclear cells of systemic lupus erythematosus patients. Cell Physiol. Biochem. 51, 979-990. doi: 10.1159/0004 95400

Tannahill, G. M., Curtis, A. M., Adamik, J., Palsson-McDermott, E. M., McGettrick, A. F., Goel, G., et al. (2013). Succinate is an inflammatory signal that induces IL-1beta through HIF-1alpha. Nature 496, 238-242. doi: 10.1038/nature 11986

Tian, F., Wang, Z., He, J., Zhang, Z., and Tan, N. (2020). 4-Octyl itaconate protects against renal fibrosis via inhibiting TGF-beta/Smad pathway, autophagy and reducing generation of reactive oxygen species. Eur. J. Pharmacol. 873:172989. doi: 10.1016/j.ejphar.2020.172989

Tsokos, G. C., Lo, M. S., Costa Reis, P., and Sullivan, K. E. (2016). New insights into the immunopathogenesis of systemic lupus erythematosus. Nat. Rev. Rheumatol. 12, 716-730. doi: 10.1038/nrrheum.2016.186

Turner, E., Liebig, J., Freiherr von, and Gregory, W. (1840). Elements of Chemistry, Including the Actual State and Prevalent Doctrines of the Science. London: Taylor and Walton. van der Poll, T., van de Veerdonk, F. L., Scicluna, B. P., and Netea, M. G. (2017). The immunopathology of sepsis and potential therapeutic targets. Nat. Rev. Immunol. 17, 407-420. doi: 10.1038/nri.2017.36

Villeneuve, N. F., Lau, A., and Zhang, D. D. (2010). Regulation of the Nrf2Keap1 antioxidant response by the ubiquitin proteasome system: an insight into cullin-ring ubiquitin ligases. Antioxid Redox. Signal. 13, 1699-1712. doi: $10.1089 /$ ars.2010.3211

Vitale, I., Manic, G., Coussens, L. M., Kroemer, G., and Galluzzi, L. (2019). Macrophages and metabolism in the tumor microenvironment. Cell Metab. 30, 36-50. doi: 10.1016/j.cmet.2019.06.001

Wang, H., Fedorov, A. A., Fedorov, E. V., Hunt, D. M., Rodgers, A., Douglas, H. L., et al. (2019). An essential bifunctional enzyme in Mycobacterium tuberculosis for itaconate dissimilation and leucine catabolism. Proc. Natl. Acad. Sci. U.S.A. 116, 15907-15913. doi: 10.1073/pnas.1906606116

Wang, Q., Li, X. L., Mei, Y., Ye, J. C., Fan, W., Cheng, G. H., et al. (2020). The anti-inflammatory drug dimethyl itaconate protects against colitis-associated colorectal cancer. J. Mol. Med. 98, 1457-1466. doi: 10.1007/s00109-020-01963-2

Weiss, J. M., Davies, L. C., Karwan, M., Ileva, L., Ozaki, M. K., Cheng, R. Y., et al. (2018). Itaconic acid mediates crosstalk between macrophage metabolism and peritoneal tumors. J. Clin. Invest. 128, 3794-3805. doi: 10.1172/JCI 99169

Weyand, C. M., and Goronzy, J. J. (2021). The immunology of rheumatoid arthritis. Nat. Immunol. 22, 10-18. doi: 10.1038/s41590-020-00816-x

Williams, N. C., and O'Neill, L. A. J. (2018). A role for the krebs cycle intermediate citrate in metabolic reprogramming in innate immunity and inflammation. Front. Immunol. 9:141. doi: 10.3389/fimmu.2018. 00141

Wu, R., Chen, F., Wang, N., Tang, D., and Kang, R. (2020). ACOD1 in immunometabolism and disease. Cell Mol. Immunol. 17, 822-833. doi: 10.1038/s41423-020-0489-5

Wynn, T. A., and Vannella, K. M. (2016). Macrophages in tissue repair, regeneration, and fibrosis. Immunity 44, 450-462. doi: 10.1016/j.immuni.2016.02.015

Xu, M., Jiang, P., Sun, H., Yuan, X., Gao, S., Guo, J., et al. (2020). Dimethyl itaconate protects against lipopolysaccharide-induced endometritis by inhibition of TLR4/NF-kappaB and activation of Nrf2/HO-1 signaling pathway in mice. Iran J. Basic Med. Sci. 23, 1239-1244. doi: 10.22038/ijbms.2020.44151.10346

Yamamoto, M., Kensler, T. W., and Motohashi, H. (2018). The KEAP1NRF2 system: a thiol-based sensor-effector apparatus for maintaining redox homeostasis. Physiol. Rev. 98, 1169-1203. doi: 10.1152/physrev.0002 3.2017

Yang, C., Liu, T., and Shi, G. P. (2020). Therapeutic potential of tricarboxylic acid cycle metabolite itaconate in cardiovascular diseases. EBioMedicine 59:102938. doi: 10.1016/j.ebiom.2020.102938

Yang, Y., Cai, X., Yang, J., Sun, X., Hu, C., Yan, Z., et al. (2014). Chemoprevention of dietary digitoflavone on colitis-associated colon tumorigenesis through inducing Nrf2 signaling pathway and inhibition of inflammation. Mol. Cancer 13:48. doi: 10.1186/1476-4598-13-48

Yi, Z., Deng, M., Scott, M. J., Fu, G., Loughran, P. A., Lei, Z., et al. (2020). Immune-responsive gene 1/itaconate activates nuclear factor Erythroid 2Related Factor 2 in hepatocytes to protect against liver ischemia-reperfusion injury. Hepatology 72, 1394-1411. doi: 10.1002/hep.31147

Yu, X. H., Zhang, D. W., Zheng, X. L., and Tang, C. K. (2019). Itaconate: an emerging determinant of inflammation in activated macrophages. Immunol. Cell Biol. 97, 134-141. doi: 10.1111/imcb.12218

Zhang, D., Lu, Z., Zhang, Z., Man, J., Guo, R., Liu, C., et al. (2019). A likely protective effect of dimethyl itaconate on cerebral ischemia/reperfusion injury. Int. Immunopharmacol. 77:105924. doi: 10.1016/j.intimp.2019.105924

Zhang, S., Jiao, Y., Li, C., Liang, X., Jia, H., Nie, Z., et al. (2021). Dimethyl itaconate alleviates the inflammatory responses of macrophages in sepsis. Inflammation 44, 549-557. doi: 10.1007/s10753-020-01352-4

Zhao, C., Jiang, P., He, Z., Yuan, X., Guo, J., Li, Y., et al. (2019). Dimethyl itaconate protects against lippolysacchride-induced mastitis in mice by activating MAPKs and Nrf2 and inhibiting NF-kappaB signaling pathways. Microb. Pathog. 133, 103541. doi: 10.1016/j.micpath.2019.05.024

Zhao, Z., Shang, Z., Vasconcelos, Z., Li, C., Jiang, Y., Zu, S., et al. (2020). Zika virus infection leads to variable defects in multiple neurological 
functions and behaviors in mice and children. Adv. Sci. 7:1901996. doi: 10.1002/advs.201901996

Zheng, Y., Chen, Z., She, C., Lin, Y., Hong, Y., Shi, L., et al. (2020). Four-octyl itaconate activates Nrf2 cascade to protect osteoblasts from hydrogen peroxide-induced oxidative injury. Cell Death Dis. 11:772. doi: 10.1038/s41419-020-02987-9

Zhu, X., Long, D., Zabalawi, M., Ingram, B., Yoza, B. K., Stacpoole, P. W., et al. (2020). Stimulating pyruvate dehydrogenase complex reduces itaconate levels and enhances TCA cycle anabolic bioenergetics in acutely inflamed monocytes. J. Leukoc. Biol. 107, 467-484. doi: 10.1002/JLB.3A111 9-236R
Conflict of Interest: The authors declare that the research was conducted in the absence of any commercial or financial relationships that could be construed as a potential conflict of interest.

Copyright (c) 2021 Lin, Ren, Gao, Dai and Yu. This is an open-access article distributed under the terms of the Creative Commons Attribution License (CC BY). The use, distribution or reproduction in other forums is permitted, provided the original author(s) and the copyright owner(s) are credited and that the original publication in this journal is cited, in accordance with accepted academic practice. No use, distribution or reproduction is permitted which does not comply with these terms. 